\title{
Flexural Pivots for Oscillatory Scanners
}

\author{
David C. Brown \\ Cambridge Technology Inc. \\ Lexington, Massachusetts, USA
}

\section{CONTENTS}

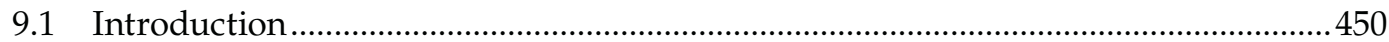

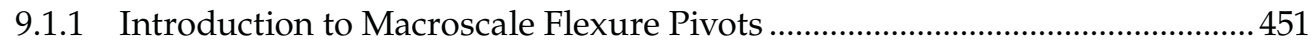

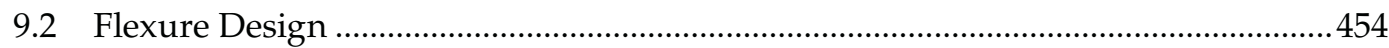

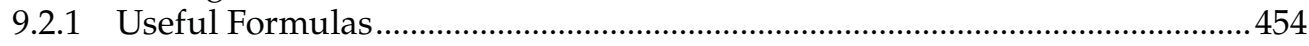

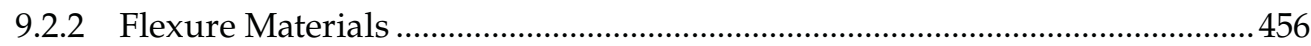

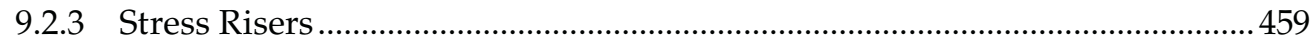

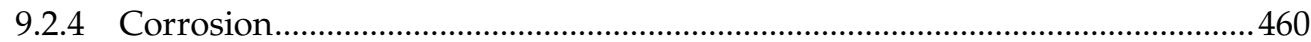

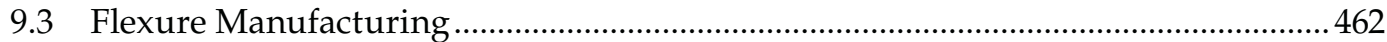

9.3.1 Manufacturing the Material.......................................................................... 462

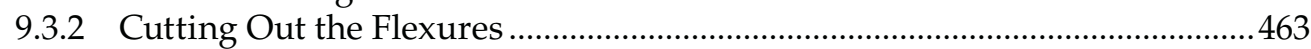

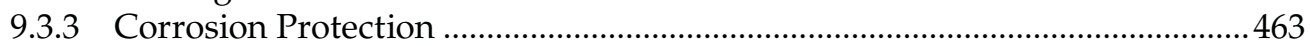

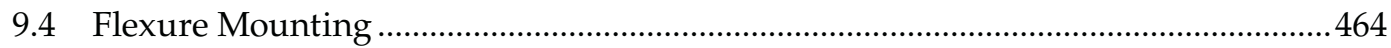

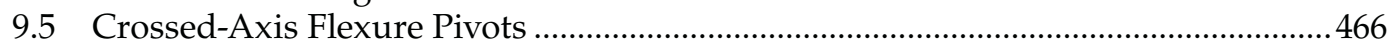

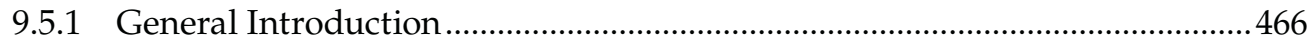

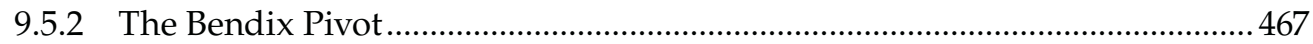

9.5.3 Cambridge Technology Crossed-Flexure Design Example ...............................468

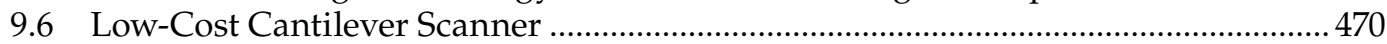

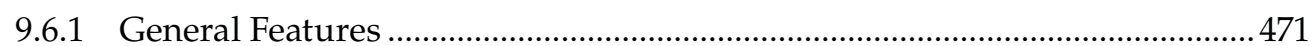

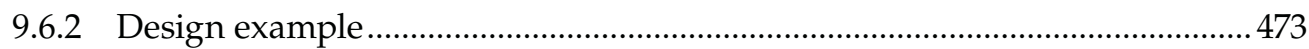

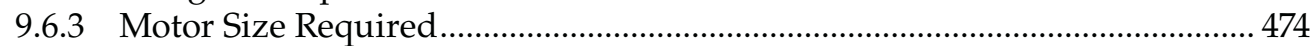

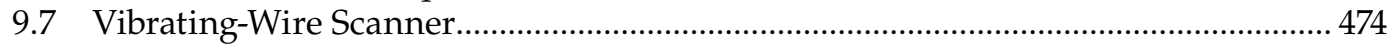

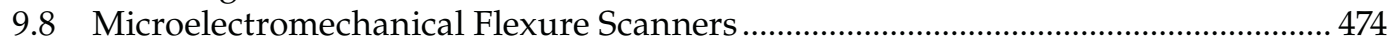

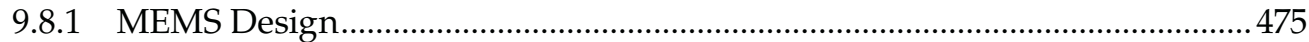

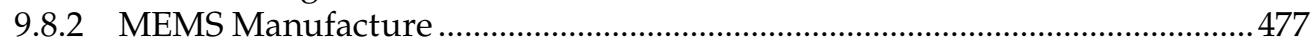

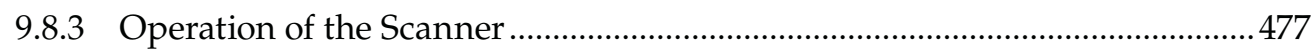

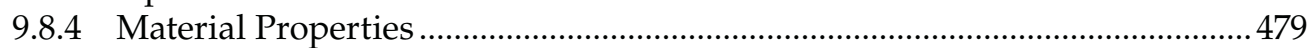

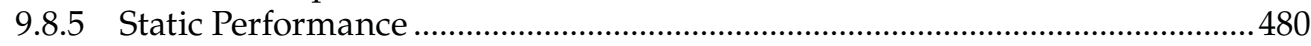

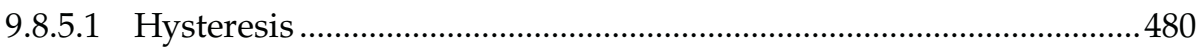

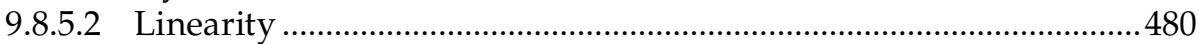

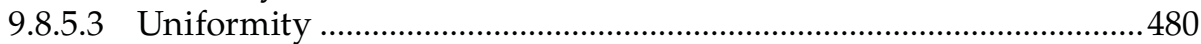

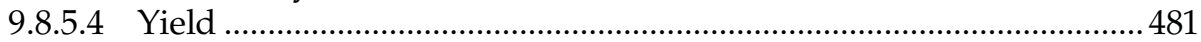

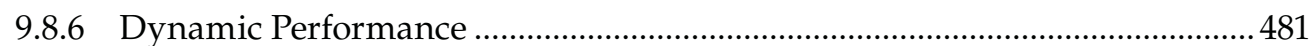

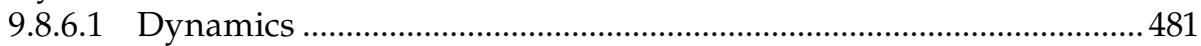

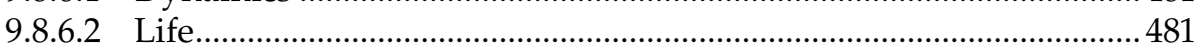


9.8.6.3 Degradation Processes ........................................................................ 481

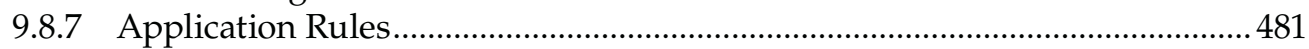

9.8.7.1 When and When Not to Use MEMS ................................................ 481

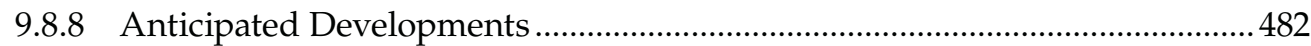

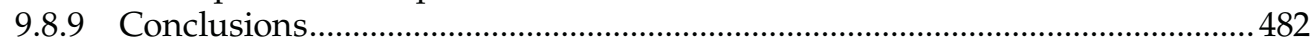

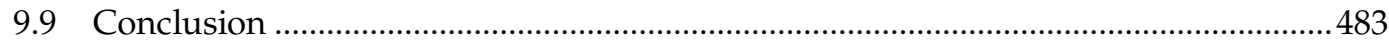

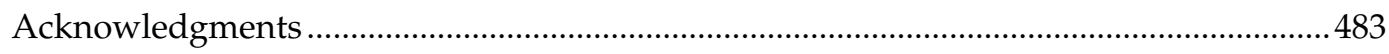

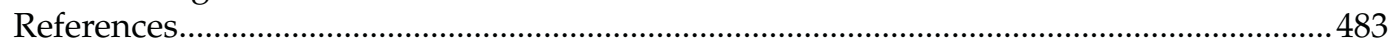

\subsection{INTRODUCTION}

The memory is the knapsack of the mind. When setting out on a journey, particularly one that promises to be long and challenging, it is better to fill it with maps and recipes, fishhooks and twine, matches and a few candles rather than tinned goods. These things are more useful, and are sure to last far longer. For those of you who cannot resist the quick fix, we hope that there are a few chocolate bars scattered about as well.

Flexures are quite ancient, and their use as pivots is also ancient. Long before the use of the most primitive bearings, leather strap flexures were used as trunk lid hinges and the like. Early war engines, for example the ballista of the Romans, the limbs of technically advanced hand bows, such as those attributed to the Turks, and the crossbows of the 14th century all employ flexures as their enabling technology.

Many pendulum clocks suspend their pendula by means of a flexure. The mechanical metronome, a specialized inverted form of clock, relies heavily on the flexure suspension in its design. It is at least arguable that the tuning fork is a pair of coupled flexures, and the music box comb is a set of flexures tuned to self-resonate at desirable frequencies.

All of these examples, and of course there are many others, exploit the flexure pivot because of its simplicity, reliability, lack of internal clearance, long service life, ease of construction, and often, its high mechanical "Q." The flexure pivots used in scientific instruments, including optical and laser scanning equipment, exploit these very same attributes.

Given the great attention devoted to flexure pivots during the 1960s and 1970s as a result of the need for rugged, reliable, light-weight, unlubricated pivots and bearings for space exploration applications, and the fact that the best and brightest were naturally attracted to what was undoubtedly the biggest science of its time, so much progress was made in flexure pivots and suspensions that one might be tempted, like Charles H. Duell, Commissioner of U.S. Office of Patents, who urged President William McKinley to abolish his office in 1899, to conclude that everything useful had already been invented.

However, as the second section of this chapter suggests, flexure technology may yet again provide one of the enabling elements in mankind's next leap of technological advancement, universal connectivity by means of light. Of course, like the flexure itself, this is an old idea, widely practised on a small scale for many centuries. The Romans communicated between the watch towers along the borders of their far-flung empire in this way, and the indigenous people of North and South America did so as well.

The organization and content of this chapter are intended to reflect the contrast between the rather settled state of macroscale flexure pivots, and the as yet entirely unexplored 
space of the microelectromechanical flexure scanners (MEMS) flexure pivot. In the case of the former, the primary author has chosen to dwell largely on the details of manufacturability, which are the fruit gained from many years spent extracting the stones from the fertile soil of the garden of flexures-the practicum, as it were, of the craft. This is the sort of information that is not generally gained through textbooks, and, in the end, differentiates a possible mechanism from a practicable, not to say elegant one. In the case of the latter, the primary author has endeavored to present the theoretical underpinnings of integrated benders, as well as the fabrication and characterization of high-speed, large-angle optical scanners constructed with them.

\subsubsection{Introduction to Macroscale Flexure Pivots}

First, let us begin by defining what we shall mean by the term "pivot."* We must begin by modifying the term "pin," because flexure pivots sometimes have virtual pins or axles. A good working definition of pivot in this context might be "A device which defines a virtual axis of rotation, over a limited angle, while fixing the other five degrees of freedom. A device which is able to resist moments except in one axis of rotation, and may be able to resist relatively small moments in that as well." In general, these pivots are of interest in instrument quality or scientific applications, as opposed to things like lorry fifth wheel applications, where they would work, but would have no particular advantages over the more common commodity sorts of bearings, such as sleeve, ball, roller, and the like.

What then are the attributes that equip flexure pivots for instrument and scientific applications? In no particular order, they are:

1. Low mass

2. Zero clearance

3. Intrinsic restoring force

4. Infinite life

5. No lubrication

6. Low hysteresis

7. No viscous losses

8. No friction (at least none in the usual sense)

9. No pariculate generation

10. No outgassing

11. No intrinsic temperature limit

12. Extremely high load capability

13. Extremely high force linearity over small angles

14. Flexibility in design configuration, in the sense that the pivot members may be pierced to permit transparency, or even to permit the passage of solid mechanical parts or objects (in contrast with conventional bearings, which are pretty much impenetrable three-dimensional solids)

15. Low design and tooling cost

16. Very low part cost

* P. 978, Chambers 20th Century Dictionary, W\&R Chambers Ltd, 43-45 Allendale street, Edinburgh EH7 4A2. 


\section{Predictability \\ 18. The ability to withstand high shock and vibration loading \\ 19. Short lead time \\ 20. Others for very specific applications}

The choice of material for a flexure is driven by conflicting requirements. For example, it is often the case that the precision of location of the pivot point is secondary to some other requirement, such as long life, low operating force, or high strength, or all the above. It may be that linearity of operating force over the desired angle of motion is also unimportant. There are often operating environment considerations. It may be that easy replacement of a damaged pivot is required. The choice of leather for trunk lid flexures results from meeting requirements such as these.

On the other hand, the designer of a bow limb has the opposite requirement that the operating force be large. Because, however, he must deal with stresses in one direction only, unlike the trunk lid flexure designer, he will exploit that difference to his advantage, and construct a flexure that is a composite. The front or tension side of his limb will be constructed from horn, which is quiet stiff in tension, while the belly, or compression side, will be constructed of sinew, which is stiff in compression. He will rightly conclude that the respective stresses in this structure are controlled by their separation from the neutral plane, and will provide a "filler" of wood properly shaped to maximize the stresses in the working "skins" of his bow without precipitating failure.

This pair of examples is designed to illustrate the broad range of potential applications of flexure pivots, and in particular the degree to which clever design is able to cope with many-order-of-magnitude differences in an application-specific parameter. Leather is able to store only a few tenths of a Joule per kilogram, whereas the best Turkish bows store over 750 Joules per kilogram, eclipsing steel and rivalling the best of the modern composites. The attribute of energy storage, related to the density, strength, and Young's modulus of the materials, may be of critical importance, required to be very high or very low, or of no importance at all in a particular application.

The absence of mechanical noise is a benefit of flexure pivots as well. Flexures are inherently low-noise pivots. Because they have no loose parts, it is unnecessary to preload them to remove clearances. As they are monolithic structures, they display very little "noise" in their force versus displacement curves. Other pivot types, comprised as they are of loose parts, make some mechanical noise during operation, and as they wear and the clearances increase, the noise level rises. Any particulates released during the wear process, of course, can cause sudden changes in the position and parasitic torque of the pivot, as well as mechanical noise, if they jam between the moving parts. Bearing "rumble" is widely recognized and even quantified as an inherent characteristic of ball bearings. In many cases, the presence of this bearing noise places an upper limit on the allowable noise bandwidth of any servo system associated with the pivot.

The possibility of a pivot design with inherently low mechanical loss is an area where flexure pivots shine. All other types of pivots known are lossy. Losses resulting from lubricant viscous friction and ball and race deformation energy not only place upper limits on the speeds at which these devices can operate, but also consume energy, limiting the mechanical efficiency of all known moving-element bearings to a low value.

Flexures, on the other hand, have no lubrication requirement, and are limited only by the internal energy of deformation. Mechanical " $Q$ " approaching 3000 is regularly achieved, even in very high speed designs. This attribute of flexures makes possible 
resonant scanners operating above $10 \mathrm{kHz}$ at very low power levels. Of course, the absence of lubrication is itself a benefit in applications in optical instrumentation, spectroscopy, space research, medicine, and semiconductor processing, where even minute contamination levels are an issue.

Flexures work extremely well for small-angle applications, where stiction, traction, surface finish limitations, mechanical tolerances, lubrication distribution requirements, and so forth put demands on other pivot types which they cannot meet successfully. Flexures are, of course, free of any looseness or play, so there is no "backlash" whatsoever associated with their use; moreover, because they rely on molecular stretching, their intrinsic hysteresis over small angles near the neutral position is always less than the unbalanced forces resulting from unavoidable asymmetries in their mountings (which produce some hysteresis in realizable designs; hysteresis levels of less than $0.1 \%$ are difficult to achieve, but possible). On the other hand, unlike other pivot types, flexures are not capable of continuous rotation. While several hundred degrees of rotation seem plausible, the authors know of no application of flexure pivots designed to operate over $90^{\circ}$, and most designs are for much smaller angles.

Other precision pivot types, such as ball bearings and jewel ("watch") bearings are limited in their geometrical precision by the limits of accuracy that their respective manufacturing processes impose on them. For example, the best class of bearing balls, class 3, has an allowed out-of-roundness of $3 \times 10^{-6}$ in. If averaged in a bearing with nine balls, the best error in "wobble" of the whole assembly will be about $9 \times 10^{-2}$ less, or about 1 $\mu$ in. If two such bearings 1 in apart support an axle, then the axle will have a wobble of about $2 \mu \mathrm{rad}$, not including errors in the concentricity of the bearing rings, axle to ring mounting, and so on. This kind of error in pivots, that is, error that is associated with geometrical features on the parts, tends to be coherent with the motion of the pivot, and so is periodic. As a result, these errors give rise, in raster scanning systems in particular, to undesirable moiré patterns. Moirés are vastly more noticeable by human observers than are nonperiodic or random errors, and can sometimes lead to rejection of a scanner that actually meets its design requirements for wobble. This effect is particularly noticeable in systems designed for printing applications, where the eye is sensitive to moirés generated by periodic angular errors in the $1 / 10 \mu \mathrm{rad}$ range. Flexure pivots have none of these sources of error. Production printing engines using flexure scanners in a raster mode built at the authors' factory regularly achieve a level of periodic error below $1 / 10 \mu \mathrm{rad}$, and were developed specifically to solve the moiré problem.

There is a tendency for conventional pivot bearings to become more fragile as the level of precision required of them is increased. This is not surprising, because the accuracy of the pivot is established by careful dimensional and geometrical control of several loose parts. The single $1 \mu$ in dent in a bearing ring, which is undetectable in a $10 \mu \mathrm{rad}$ application, becomes the limiting factor in a $1 \mu \mathrm{rad}$ application. Flexures, on the other hand, are extremely robust, impervious to dust and other mechanical and most chemical contamination, and are very insensitive to shock and vibration. In most cases, a flexure pivot will survive indefinitely in a factory-floor or outdoor application in which other pivot types have very limited service life, if they operate at all.

Lastly, flexure pivots have inherently infinite life if operated at a peak stress level below the fatigue limit for the material from which they are made. Some care is required in eliminating any sort of stress riser in the design, and also in determining the effective fatigue limit for the material. Cambridge Technology (Lexington Massachusetts) regularly warrants its flexure products for 5 years of operation in any environment, at any duty cycle, with no statistically significant failure rate. For an $8-\mathrm{kHz}$ scanner operated continuously, this amounts to more than $10^{12}$ cycles. (Of course, ball bearings won't operate at $8 \mathrm{kHz}$ in 
an oscillatory mode. However, by contrast, in applications below $1 \mathrm{kHz}$ where they will operate satisfactorily, their life is generally limited to between 1 and $5 \times 10^{* 9}$ cycles.)

Of course, flexure pivots are not perfect. In general, the transverse stiffness of the flexure pivot is inferior to that achievable with ball bearings, resulting in unintended cross-axis motions in the presence of significant environmental stimuli, or in gyroscopic reaction to very large accelerations of the axle. It is also more difficult to achieve very large scanning angles with flexure pivots, for the reason that the curl of the flexures at extreme angles reduces even further their transverse stiffness. The allowable stress limit at the extreme angle legislates in favor of quite thin flexures, further reducing their stiffness. While flexure scanners with scan angles up to $80^{\circ}$ optical have been produced, their tolerance of environmental vibration and axle acceleration is low because of these effects, particularly when equipped with the very thin flexures required to obtain extremely long lifetimes.

Flexure pivots generally are designed to constrain the minimum number of degrees of freedom required by the application. Often, for example, a translation of the axle parallel to its axis is allowed, although even when the translation is not obnoxious optically, it has the potential to set up undesirable vibrations, and, in the limit, catastrophic mechanical positive feedback. With crossed-flexure pivots, it is possible to construct a geometry with zero axle shift, and practical to expect such a design to produce translation of the axis of less than a few microns over a small angle of rotation.

\subsection{FLEXURE DESIGN}

The possible combinations and permutations of flexures in a design approach for an arbitrary application is so vast that no attempt will be made here to cover any but the simplest form of flexure, the single leg. In the case of symmetrical designs, the easiest approach is to multiply the width of a single leg by the total number of flexures to model the flexure as a unit. In the case of asymmetric designs, other approaches to fit the circumstances will require invention. The formulas below in Section 9.2.1 assume fully reversed stress; that is, that the flexure is bent symmetrically through the same angle on both sides of the neutral position. As in the case of the Turkish bow, unreversed, or partially reversed stress provides an opportunity for clever design and perhaps nonmonolithic flexure materials. In fact, the definition of "fatigue limit" as it is used here, "That stress which represents the maximum stress which will allow the flexure to operate indefinitely without failure under a particular set of circumstances," is a hotly contested topic, and those who believe they have a suitable answer guard their secret with the most intense passion. The case of the partially reversed stress is an even more volatile subject. Obviously one of the corner stones of flexure design is an understanding of the safe upper limit of flexure stress. In the absence of reliable published data, and for the purpose of designing for a standard laboratory environment, it is the considered opinion of the authors that, for ferrous material, a value of $35 \%$ of the ultimate strength is safe under dry operating conditions.

\subsubsection{Useful Formulas}

The primary parameters of interest to a flexure pivot designer include the rotational spring rate of the pivot, the maximum scan angle achievable under some maximum stress level, the maximum stress applied to a flexure bent through some scan angle, the allowable 
thickness for a flexure whose fatigue limit, length, Young's modulus, and angle of operation are known, and the first resonant frequency of the axle-pivot assembly.

It will be obvious that these formulas are a first-order approximation. It has been found, however, that the errors produced are small enough so that minor variations in the density of the materials used, the actual effective length and thickness of the flexures, and so on, dominate the result, and any application that requires very precise control of a particular parameter, such as the frequency of a mechanical resonator, will require some "tweaking" mechanism for final tuning on an individual basis.

These formulas also assume that the flexure mountings are infinitely rigid, support the flexure completely and uniformly, introduce no stress risers, and allow no relative motion. Except for the rigidity, these conditions are usually met satisfactorily by careful design. The first-time designer would be well advised to do a careful finite element analysis (FEA) of the mounting rigidity, or allow for an iteration or two in the design schedule. The formulas are given as follows for constant-section flat springs:

\section{For Crossed-Flexure Pivots:}

Rotational spring rate

$$
K=E W T * 3 / 12 L
$$

Peak mechanical angle in radians

$$
A=\frac{2 L S}{E T}
$$

Maximum stress

$$
S=\frac{E T A}{2 L}
$$

Thickness

$$
T=\frac{2 L S}{E A}
$$

\section{For Cantilever Flexure Pivots}

$K=P / d=3 E I / L^{* 3}=W T^{*} 3 E / 4 L^{* 3}$

$S=6 P L / W T^{*} 2$

$A=d / L$

$P=K d$

$d=A L$

$T=(6 P L / W S)^{*} 1 / 2$

$U=1 / 2 d^{*} 2$

Resonant frequency

$$
F=1 / 2 p(K / J) * 1 / 2
$$


where $E$ is Young's modulus of the flexure material, $W$ is the width of the flexure, $T$ is the thickness of the flexure, $L$ is the effective active length of the flexure, $A$ is the peak scan angle of the flexure in radians, $S$ is the peak stress in the flexure, $P$ is the load at the tip of the cantilever, $d$ is the deflection at the tip of the cantilever, and $F$ is the first rotational resonant frequency of the flexure/axle system or the fundamental vibrational frequency of the cantilever/mirror system, where $J$ is the combined moment of inertia of the respective system. Of course, use of consistent units is required.

There is an extensive bibliography on the subject of detailed design of flexure pivots, of which several are listed in the reference section.

\subsubsection{Flexure Materials}

It will be noticed immediately from inspection of the relationships among the physical constants pertinent to flexure materials that the stress is directly proportional to Young's modulus. The allowable stress is proportional to the fatigue limit of the flexure material, which most workers agree is itself proportional to the ultimate strength of the material. (Since it is now generally agreed that the so-called proportional limit, or yield strength, is not a useful concept, we shall avoid using it here.) The natural conclusion from this train of reasoning is that one could rank flexure materials by a figure of merit that is the result of dividing the material's fatigue limit by its Young's modulus. Because the exact value of the fatigue limit is unknown, or at least unpublished for the materials of interest, we shall use the ultimate strength instead, since the two are believed to be directly proportional.

Leaving for those who follow us those exotic materials whose currently available form or lack of observable malleability or other defect make their use questionable, we can construct Table 9.1.

There are some applications in which weight, or minimizing it, are paramount. In this case, specific strength, rather than ultimate strength, can be used in the construction of a figure of merit list, which might look like Table 9.2.

As one would expect, aluminum and titanium have moved up the list, but little else has changed. One could, of course, construct other lists for other specific purposes, such as minimization of inertia. Since the inertia of the flexures themselves is rarely significant construction of this list is left to the interested reader. The units of inertia are mass $x$ radius squared, so dividing specific strength by dynamic stiffness would produce such a list (dynamic stiffness is Young's modulus divided by density squared to account for the radius squared term).

However, there are two caveats. First, most engineering enterprises are constrained by commercial goals, and so the use of exotic materials is quite properly frowned upon unless there is robust technical or other justification for their use. To the knowledge of the authors, precious metal alloys such as BeAu and spring gold are used almost exclusively in the manufacture of very high quality fountain pen nibs. In this application, they are justified because the unique combination of fatigue resistance, corrosion resistance, wear resistance, appearance, and "feel" enhance the marketability of the end product. Secondly, the fact is that very little is actually known, or at least published, about the long-term fatigue resistance of nonferrous alloys. This is partly the result of the fact that ferrous alloys have been in use for a very long time, and their overall combination of characteristics have recommended them for so many demanding applications that they are both well studied and in ready supply. One could argue that copper alloys have been in use longer, and while that is true, these alloys were generally eclipsed by their ferrous brethren as soon as they 
TABLE 9.1

Parameters for Various Materials, Including Ultimate Strength

\begin{tabular}{llcc}
\hline Material & $\begin{array}{c}\text { Young's } \\
\text { Modulus (psi) }\end{array}$ & Ultimate Strength (psi) & Ratio \\
\hline Carbon/graphite & $<2 \times 10^{6}$ & $375 \times 10^{3}$ & 0.19 \\
Diamond & $150 \times 10^{6}$ & $7.69 \times 10^{6}$ & 0.051 \\
Glass reinf. epoxy & $5 \times 10^{6}$ & $240 \times 10^{3}$ & $0.05^{\mathrm{a}}$ \\
Silicon & $27.5 \times 10^{6}$ & $1.02 \times 10^{6}$ & 0.037 \\
$\mathrm{Be} / \mathrm{Au}$ & $15 \times 10^{6}$ & $210 \times 10^{3}$ & 0.014 \\
Spring gold & $15 \times 10^{6}$ & $180 \times 10^{3}$ & 0.012 \\
Be/Cu & $18 \times 10^{6}$ & $180 \times 10^{3}$ & $0.01^{\mathrm{b}}$ \\
Ti ${ }^{-6} \mathrm{Al}{ }^{-4} \mathrm{~V}$ & $19 \times 10^{6}$ & $205 \times 10^{3}$ & 0.01 \\
$7075 \mathrm{Al}$ & $10 \times 10^{6}$ & $98 \times 10^{3}$ & 0.009 \\
Udeholm 718 & $30 \times 10^{6}$ & $265 \times 10^{3}$ & 0.009 \\
$17-7 \mathrm{PH}$ & $30 \times 10^{6}$ & $235 \times 10^{3}$ & 0.008 \\
Inconel & $31 \times 10^{6}$ & $250 \times 10^{3}$ & 0.008 \\
$302 \mathrm{SS}$ & $28 \times 10^{6}$ & $200 \times 10^{3}$ & $0.007^{\mathrm{c}}$ \\
\hline
\end{tabular}

a This material's lack of electrical conductivity may limit its usefulness.

b $\mathrm{Be} / \mathrm{Cu}$ has published figures of fatigue limit of $40 \mathrm{ksi}^{1}$

c Interestingly, this material not only work hardens, but its Young's modulus rises with work as well. One should, therefore, try to get material rolled as thoroughly as possible, so that the rise in modulus during use will be minimized. The range of moduli published are $24-28 \times 10^{6} \mathrm{psi}^{1}$

TABLE 9.2

Parameters for Various Materials, Including Specific Strength

\begin{tabular}{lccc}
\hline Material & Young's Modulus (psi) & Specific Strength (psi) & Ratio \\
\hline Carbon/graphite & $<2 \times 10^{6}$ & $3509 \times 10^{3}$ & 1.75 \\
Glass reinf. epoxy & $5 \times 10^{6}$ & $3200 \times 10^{3}$ & $0.64^{\mathrm{a}}$ \\
Silicon & $27.5 \times 10^{6}$ & $12300 \times 10^{3}$ & 0.448 \\
Diamond & $150 \times 10^{6}$ & $61000 \times 10^{6}$ & 0.407 \\
$7075 \mathrm{Al}$ & $10 \times 10^{6}$ & $961 \times 10^{3}$ & 0.10 \\
$\mathrm{Ti}^{-6} \mathrm{Al}^{-4} \mathrm{~V}$ & $18.5 \times 10^{6}$ & $1120 \times 10^{3}$ & 0.06 \\
$\mathrm{Be} / \mathrm{Cu}$ & $18 \times 10^{6}$ & $557 \times 10^{3}$ & $0.03^{\mathrm{b}}$ \\
$\mathrm{Udeholm} 718$ & $30 \times 10^{6}$ & $936 \times 10^{3}$ & 0.03 \\
$17-7 \mathrm{PH}$ & $30 \times 10^{6}$ & $830 \times 10^{3}$ & 0.03 \\
Be/Au & $15 \times 10^{6}$ & $301 \times 10^{3}$ & 0.02 \\
Spring gold & $15 \times 10^{6}$ & $301 \times 10^{3}$ & 0.02 \\
Inconel & $31 \times 10^{6}$ & $749 \times 10^{3}$ & 0.02 \\
302SS & $28 \times 10^{6}$ & $697 \times 10^{3}$ & $0.02^{\mathrm{c}}$ \\
\hline
\end{tabular}

a This material's lack of electrical conductivity may limit its usefulness.

b $\mathrm{Be} / \mathrm{Cu}$ has published figures of fatigue limit of $40 \mathrm{ksi}^{1}$

c Interestingly, this material not only work hardens, but its Young's modulus rises with work as well. One should, therefore, try to get material rolled as thoroughly as possible, so that the rise in modulus during use will be minimized. The range of moduli published are $24-28 \times 10^{6}$ psi. $^{1}$ 
became available. The stainless steels have replaced bronzes where corrosion resistance is important, and $\mathrm{BeCu}$ and phosphor bronze for springs except for applications where magnetic susceptibility or electrical or thermal conductivity are issues.

In fact, it seems from the published literature on the subject that nonferrous metals have disappointingly low fatigue limits. ${ }^{1}$ Experimental work at Cambridge Technology with $\mathrm{BeCu}$ flexure materials reinforces this view.

However, the primary reason for their use is probably simply that ferrous materials make very good flexures, and except for very unusual circumstances, the apparent technical superiority of the "other" material, if actually realizable, would require the investment of such large development or other resources that return on the investment might well be negative. As a case in point, Cambridge Technology has abandoned the "stress-proof" steels, number 8 on the list, in favor of 302SS, which is last in both Tables 9.1 and 9.2. While the manufacturer of air compressors has available to him thicknesses of material appropriate to his flapper-valve needs, no one stocks the thin foils needed for flexures. This material cannot be rerolled, and so a new thickness requirement calls for a mill run minimum at a cost of $\$ 25,000$ or more and 2 years wait, and which yields a supply of material which is vastly in excess of the needs of any flexure product lifetime yet known, and must be stored carefully, inventoried, and so forth for many, many years against possible future demand, or else expensed and discarded.

Of course, not all pivots are required to last indefinitely. It is likely that an exploratory scientific mission to Mars, for example, might require only a few hundred thousand actuations. In circumstances such as these, since low weight and high reliability are much more important than life, it is probable that resources can be saved by designing a limited-life flexure. It is easy to think of other applications in which this may be true, and the author designed and flew successfully flexure systems required to operate only a few tens of cycles during atmospheric testing of atomic weapons.

Unfortunately, it appears that the borderline between indefinite life and finite life is clifflike. It is fairly easy to predict accurately life versus stress for small to moderate cycle lives, because testing to validate the model is practical. As an example, the Bendix Corporation (Utica, NY) published graphs ${ }^{2}$ showing life versus cycles for their standard flex pivots under various conditions of loading. These graphs show load lines at 35,000 cycles, 220,000 cycles, and indefinite life. These curves cover a factor of 2 in angle, and $0 \%-100 \%$ loading. Interpolation is possible, but as one approaches indefinite life, small errors in actual loading and angle can cause one to fall off the cliff, with disastrous results. Testing a device to demonstrate "indefinite" life takes a very long time, and various forms of accelerated testing should be approached with caution. That said, many manufacturers of flexures have developed accelerated testing methodology. They, like Cambridge Technology, protect these methods as trade secrets. A rule of thumb, in keeping with the most widely accepted theory of fatigue failure, is that a flexure design that passes $3 \times 10^{7}$ cycles without failure is destined to operate indefinitely in a dry environment at this or lower level of stress. ${ }^{3}$

As the next section discusses, there are some hidden requirements for the qualities of the raw material form that should be understood before extravagant expense is lavished on the development of a material whose soundness of internal structure and finishability are questionable. That said, whenever an exotic, hard-to-work, expensive, material has significant technical justification, then it is worth exploring. The example of the folks who succeeded in bringing diamond phonograph styli to the mass market should be firmly held in mind. 


\subsubsection{Stress Risers}

If the failures of flexures were ranked by cause, it would be found that, except for instances that resulted from exceeding the design stress, all failures resulted from either stress risers or corrosion or a combination. The next section deals with the germane corrosion issues. Here we will deal with the problem of stress risers, which generally arise from poor finish on the surface of the flexure foil, nicks in the edges of the flexure as manufactured, mounting defects, or inclusions in the flexure material itself, in about that order of importance. The sections on flexure manufacturing and flexure mounting deal in some detail with the avoidance of stress risers resulting respectively from manufacturing and from mounting.

A stress riser is anything that raises the stress in the flexure, either locally or globally, above the value that the designer intended that it have at that point. Because most flexures of interest to this discussion are made from rolled material, it is important to note that such materials are not really isotropic in their mechanical characteristics, the "grain" having been elongated more in one direction (the direction perpendicular to the axis of the rolls) than the other; in other words, the grain is longer in the length direction than it is in the width direction. As a result, the fatigue resistance of the material is anisotropic as well, being higher across the grain than it is parallel to the grain. It is therefore necessary to specify the direction of the grain when flexures are produced from the sheet of foil, and if the as-rolled coil is cut up into smaller sheets, the direction of rolling must be marked on each sheet in order to preserve the grain direction information. Since fatigue resistance is paramount in flexures, it is usual to specify that the flexure be cut out of the sheet in such a way as to cause the bending in operation to be across the grain.

The stress risers most prevalent in oriented flexures are the result of scratches in the surfaces of the rollers used to produce the foil. While it may be nonintuitive, the flexure stress is increased in the regions where the thickness is greater. The best quality rolled finish obtainable is none too good for flexure material, particularly when the flexure is thin. For example, a flexure 0.001 in thick, made from material with a standard mill finish of $32 \mu$ in roughness average (RA), is allowed to have scratches on both sides that are up to 0.0001 in deep. If two of them lie on top of each other, then the local thickness of the flexure is 0.0008 in. If the scratches extend to the edge of the stressed part of the flexure, the $20 \%$ reduction in area is very likely to act like a notch and induce a crack. On the other hand, if the rolls which made the foil have scratches, the foil will have inverted scratches, that is raised areas which have the effect of increasing the thickness locally. Using the same exemplar dimensions, because the stress is proportional to the cube of the thickness, the local stress will exceed the designer's intent by $95 \%$, and is likely to cause failure. Of course, an inclusion of microscopic size will produce the same effect.

Cambridge Technology specifies 4RA or better finish for its rolled flexure strip. For critical applications, it is well to have the rolled sheet $100 \%$ X-rayed to find any inclusions or cracks or voids or other defects inside the material.

The last important item is not associated with the quality of the raw material, but is one of the most easily overlooked elements of a stress riser free design. Nearly every flexure has one or more radical changes in width, usually associated with attachment. It is absolutely critical that these section changes are made with a stress-distributing fillet or radius in the corner. Otherwise, failure cracks will inevitably originate at these locations.

Figure 9.1 is a drawing of one of the flexure assemblies in current production at Cambridge Technology. This part is actually two flexures joined together by a bar (the horizontal part along datum " $\mathrm{B}$ "). Making a pair of flexures this way ensures the utmost in symmetry, since they are made at the same time from nearby regions of the same piece of metal. Also, 
Notes:

1. Material: stainless steel, type 301 or 302 , full hard condition. surface quality 4RA (rahns 2n, photoetch quality).

2. Finish: Passivate, per QQ-P-35.

3. Photoetch attachment points along datum B only

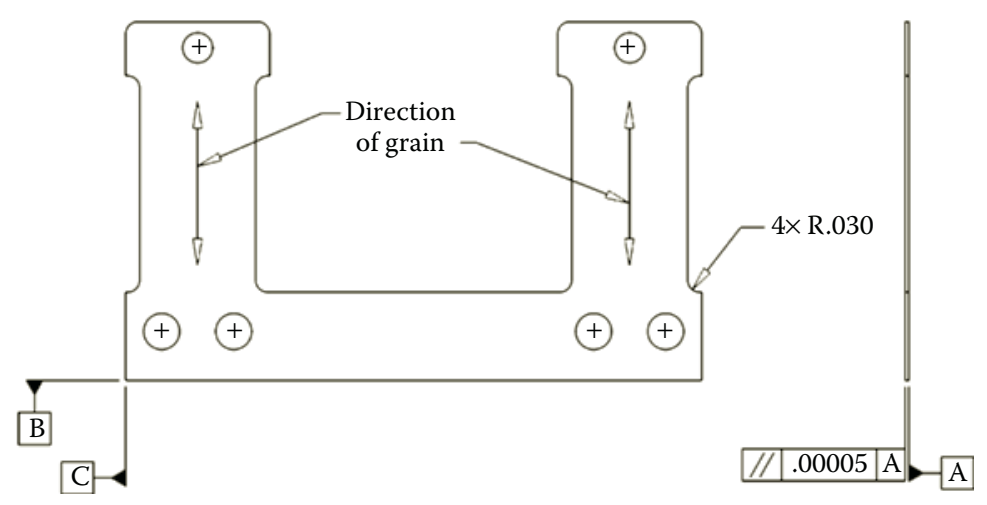

FIGURE 9.1

Typical flexure.

the "self-fixturing" feature of the bar eliminates the necessity for adjusting their parallelism and individual effective lengths during assembly.

Attention should be paid to the specification legend in the upper left-hand corner, as well as to the grain-defining arrows and the corner radii at every section change.

\subsubsection{Corrosion}

It is assumed here that the flexure designer will take into account the environment in which the equipment will live, and take suitable precautions against atmospheric or environment corrosion. We will discuss here only the forms of corrosion that result from the highly stressed conditions of the flexures during use, the effects of galvanic couples, and the hydrogen embrittlement associated with electroplating.

Corrosion in flexures is intimately connected with stress risers, because most of the corrosion effects encountered begin in cracks and crevices either caused by stress cracking or enhancing to stress cracking. However, there is one area of corrosion difficulty encountered that is not directly associated with stress, but is instead a form of electrolytic corrosion resulting from the contact between metals far enough apart on the electromotive series. This effect is not significant in very dry conditions, but the presence of an electrolyte can cause rapid erosion of the anodic member of the couple. Even atmospheric water held in cracks by capillary action can be a powerful electrolyte under conditions of stress. It is up to the designer to decide which member of the couple he would prefer to be dissolved; in general, it is preferable to protect both to the extent possible. Theoretic galvanic couples are presented in Table 9.3.

In general, MIL-STD-186 allows adjacent groups, or, in some cases, metals two groups away from each other to be coupled. This does not mean that galvanic action does not take place. It just means that for most purposes the rate of corrosion is so slow as to be unimportant.

At Cambridge Technology, standard product joins group 5 flexures with group 14 mounting materials using group 13 fasteners with satisfactory results. Because the aluminum is 
TABLE 9.3

Theoretical Galvanic Couples

\begin{tabular}{lll}
\hline Group & \multicolumn{1}{c}{ Material } & \multicolumn{1}{c}{ EMF with Respect to Calomel } \\
in Sea Water
\end{tabular}

quite anodic to the flexures, it is slowly dissolved, but the more sensitive flexures are unaffected. For demanding applications, all the ferrous parts are tin plated before assembly, and the aluminum is anodized.

In this context, it is useful to discuss a side effect of electroplating of high-strength steel, the so-called hydrogen embrittlement. During electroplating, the electrolyte, if aqueous, contains hydrogen ions, which are hydrogen atoms stripped of their electrons. These very small ions are able to squeeze into the grain boundary lattice structure of the steel, where they can produce pressures approaching 13,000 atmospheres. Under conditions of stress, the combined forces of the hydrogen and the external stress rupture the metal. It is one of the cruel jokes of nature that the very high strength steels such as those used for flexures are severely afflicted by this process, and that the heat treatment required to drive out the hydrogen severely limits the strength that can be obtained in precipitation-hardened stainless steels such as $17-4 \mathrm{PH}$. This is one of the reasons for choosing an age-hardening grade of steel such as 302 for flexures that might require electoprocessing.

The theory of stress-corrosion cracking favored by the authors is the electrochemical theory. ${ }^{4}$ According to electrochemical theory, galvanic cells are set up between metal grains and anodic paths are established by heterogeneous phases. For example, the precipitation of $\mathrm{CuAl}_{2}$ from an $\mathrm{Al}-4 \mathrm{Cu}$ alloy along grain boundaries produces copper-depleted paths in the edges of the grains. When the alloy, stressed in tension, is exposed to a corrosive environment, the ensuing localized electrochemical dissolution of the metal, combined with plastic deformation, opens up a crack in the metal. Supporting this theory is the existence of a measurable potential in the metal at grain boundaries, which is negative with respect to the potential of the grains. Once the crack exists, capillary forces aid the delivery of electrolyte to the tip of the crack, and it propagates. Figure 9.2 is an illustration of this concept. 


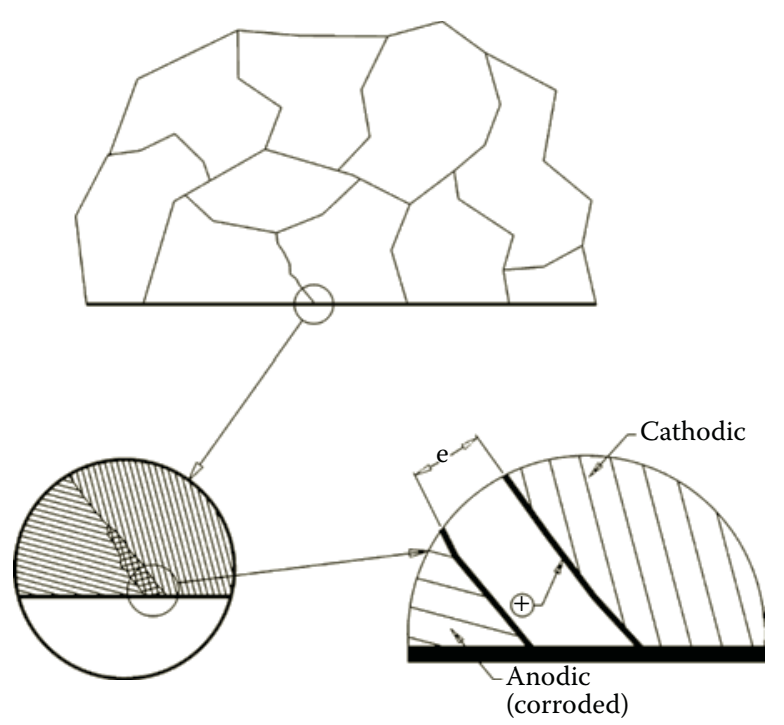

FIGURE 9.2

Stress-corrosion cracking.

\subsection{FLEXURE MANUFACTURING}

\subsubsection{Manufacturing the Material}

Flexures have been manufactured by every conceivable process, but for the purposes of small precision pivots, carefully rolled sheet or foil produces the best densification, uniformity of metallurgy, and surface finish. In addition, many work-hardenable materials come off the rolls with the proper temper for use. It cannot be overemphasized that surface finish is critical to the performance of thin flexures. No process now known is able to improve over the uniformity of thickness, flatness, freedom from scratches, and the consistency of temper of ferrous materials precision rolled on machines specially designed and constructed for the purpose. However tempting it may be to obtain experimental stock by grinding and polishing or etching down existing material, whether chemically or in combination with ion impact, these processes will change the performance values of the material. Unless one is prepared to make all the required flexures by an identical process stream, likely extremely uneconomical, the experimental flexures should be made from a material process exactly as the production flexures will be processed. In fact, considering that 100 pounds of flexure material such as type 302 stainless can be obtained as a minimum run from a reputable reroller for under $\$ 3000$, and $100 \mathrm{lb}$ of foil will make many tens of thousands of flexures, there is hardly any reason not to make the experimental parts from the production material, and there are several reasons why this is the best approach.

First, the process of arriving at the desired thickness, for the sake of argument 0.00500 in thick \pm 0.000020 in, at the required as-rolled temper, usually requires that the reroller start with carefully annealed material whose thickness is the nearest standard thickness above ten times the finished thickness, in this case, probably 1/16 in. Even so, the finished temper of the material will vary by a few percent run-to-run, the thickness will vary by 10 to 
20 millionths run-to-run, and the cross-sheet crown will also vary by a few millionths run-to-run. As a result, even if the edges of the sheet are trimmed off the material used for flexures, which is good practice, for the best uniformity flexure-to-flexure, it is best to buy all the material needed for the product lifetime in one mill run. If this is not possible, or if product demand exceeds all expectations, it is best to requalify each succeeding flexure material run, and to make adjustments in the flexure width to compensate for any variations in modulus of elasticity or spring constant discovered. The stiffness of the flexure is linear with width, and a new photo tool is only a few hundred dollars, and so adjusting the width is a reliable and economical way to titrate the flexure stiffness.

It is also worth the expense, for critical applications at least, to $100 \% \mathrm{X}$-ray the material to find any inclusions, cracks, or voids.

\subsubsection{Cutting Out the Flexures}

Flexures may be cut out of sheet or foil by punching, conventional machining of stacked blanks, laser, water jet, or E-beam cutting, or chemical etching of photolithographically produced patterns in photo resist. The last process is the standard process at Cambridge Technology, and is that preferred by most workers under most circumstances, because it is universally applicable, widely available, accurate to microinch tolerances, repeatable, fast, localized to the area to be cut, the photoresist protects the sensitive surfaces, and the process is inexpensive.

The other methods cause, or can cause, performance variation as the result of modified metallurgy in the zone near the cut. These potential modifications include heat effects, work hardening, and changes in the composition or phase of the alloy. Changes in the thickness of the flexure locally, and the introduction of stress risers, invariably accompany the relatively large-scale, relatively uncontrolled motions of mechanical machining and punching, including fine blanking, and are also associated with jets of abrasive or abrasive in water, laser cutting, and E-beam cutting.

It is also difficult to provide adequate surface protection during processing by these methods.

Even photoetching produces artifacts that have the potential for undesirable effects. For example, the edge pattern left by double-sided etching is a double cusp that is irregular, sharp, and full of stress risers. Figure 9.3 shows such an edge.

Also, the position of the attachment points between the flexures to be and the parent sheet should be specified in such a place, usually on an unstressed tie bar or mounting tab, that the stresses associated with their disconnect are not obnoxious. The best way to remove the cusps and their associated stress risers is by tumbling in appropriate media, chosen to batter rather than to scratch, so that the cusps are beaten down, but the surfaces are not scratched. The edges of the flexures should be $100 \%$ inspected under suitable magnification, and any flexure with a nick or scratch visible at $20 \times$ should be rejected.

\subsubsection{Corrosion Protection}

Most of the materials usually chosen for flexure material have intrinsic resistance to laboratory or office environments which is adequate protection. However, in shop floor, marine, outdoor, or specialized applications, further protection is often desirable. Such protections include sealing and purging the entire mechanism. When this approach is not practical, then the flexures themselves (and, if necessary, their mated parts) can be protected. Of course, with the flexures in particular, the protection should not interfere any more 
Notes:

1. Photoetch attachment points along datum B only.

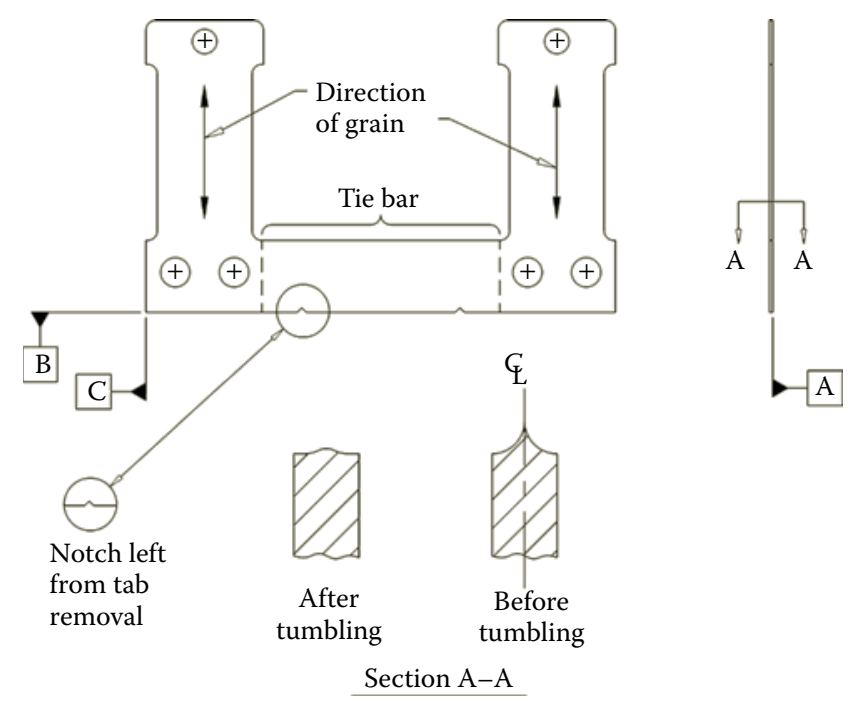

FIGURE 9.3

Edge stress risers.

than necessary with the underlying functional performance of the part. At Cambridge Technology, hot-oil reflowed tin plating of the ferrous parts, including clamps and screws, is the protection method used. Tin has excellent protection in very thin layers, is anodic to the type 302 flexure material, has a very small Young's modulus, is a lubricating film, helps to distribute the clamping forces uniformly, and has low mass. It is, however, cathodic to 5, 6, and 7000 series aluminum, as well as type 355 and 356 cast aluminum. It is, of course, extremely cathodic to magnesium and its alloys. The answer here is anodizing of any aluminum or magnesium mated parts, brought to completion, and sealed with a suitable material such as sodium silicate. Although MIL-STD-186 allows tin in contact with magnesium, the best practice is anodization of the magnesium, or, in the case of fairly porous castings, vacuum impregnation of the casting with epoxy or equivalent followed by anodization.

\subsection{FLEXURE MOUNTING}

The careful mounting of flexures is essential if the full potential of careful flexure design is to be reached. There are three aspects of mounting that deserve thought. The first is the transition from the flexure itself to the mounting provision for that flexure, whatever it may be. For example, flexures may have integral mounting tabs intended to be supported with reenforcing loose clamps, clamps attached by adhesive or solder to the flexure tab, flexures without tabs intended to be clamped or soldered or cemented into a slot, flexures with or without tabs intended to be welded in place, and so on. Whatever the method used, it is essential that the distribution of stress from the highly stressed operating region to the 
mount is gradual, controlled, and without stress risers. In general, neither the mounting area nor the body of the mount can be made to carry the concentrated stress loads that the flexure itself carries. Typically, the flexure is flared out in the region of the mounting so that the stressed region is increased in cross-sectional area, and the intensity of the stress is reduced thereby to a level low enough to prevent fracture of the mounting region before the main flexure. While any of the above methods may be used, the standard practice at Cambridge Technology is to produce a flared tab design similar to that in Figures 9.1 and 9.2. The corner radii are essential to achieving a transition without a stress raiser in the corner.

Of course, the presence of a radius in the corner raises the question of just where the effective region ends, and where the mounting region begins. It has been found by experiment that if the effective length of the flexure is taken to be the distance between the points of tangency between the constant-width section of the flexure and the radii at the ends, plus one radius, the error in the result will be dominated by tolerances on the flexure thickness.

Secondly, alignment of the flexure(s) to the stationary and moving members must be thought out. It is often the case that a multiplicity of flexure pairs or sets positioned along the axis of rotation is required in order to resist the cross-axis moments of the system. Thus, as many as four or six individual flexures may require individual alignment in a typical system. In this case, it is convenient to manufacture the flexures attached together by means of a tie bar, which may either be left attached or removed after assembly. This reduces the number of alignments required by at least two. Such a tie bar is illustrated in Figure 9.3.

Thirdly, the designer must decide whether or not to try to make the flexure mountings on the fixed or on the moving or both elements indefinitely stiff. If he succeeds, then the design formulas given earlier will produce predicable results. If not, then some iteration may be required before satisfactory performance is obtained.

However, before leaping to a conclusion here, the designer should take into consideration the fact that the economical materials of choice for the rotor and/or stator are often not high fatigue strength materials, and may not even be highly qualified materials and so may have relatively loose, or unknown specifications of physical parameters such as Young's modulus and strength. In addition, it may well be that some other parameter is of more importance to their overall function than fatigue strength. One might posit thermal conductivity for the stator, and low inertia for the rotor. Whatever the reason, it is often desirable to allow the flexure-mated parts to be made of less intrinsically stiff material than is the material of the flexure itself. One then has the option of increasing the cross-sectional area of the mounting region appropriately. However, if the reason for using, say, an aluminum-magnesium alloy for the rotor had to do with inertial minimization, then bulking up the extreme-from-the-axis parts (where the flexure ends inevitably attach) to increase their stiffness is not likely to find favor. It may, in fact, be a better overall solution to allow the mountings to bend a little under load, and recalculate the flexures, if necessary, to compensate. It should be recognized that some deflection of the flexure mountings is required anyway, because if they are as stiff as or stiffer than the flexures, the clamping loads will be at least partially transferred to the flexures, potentially overloading them at the worst possible location, the mounting transition region. In this context, it is well to pay as much attention to the flatness and surface finish of the flexure-contact pads as to that of the flexures themselves. For this reason, slots are discouraged because good surface finish on the sides of slots is difficult to achieve, and inspecting them is next to impossible. A carefully machined and lapped 
pad, with a loose, lapped clamping plate under the screw heads to distribute their local loading is much better, and less costly in the long run. A layer of tin or indium foil or adhesive between the flexure and its mating surface on each side is advised as a method of filling microscopic voids and the valleys between the peaks on the surfaces, as well as providing corrosion protection at the interfacial joints. Care in mounting flexures cannot be overemphasized, particularly when attempts have been made to make the mating structures extremely stiff.

\subsection{CROSSED-AXIS FLEXURE PIVOTS}

\subsubsection{General Introduction}

Of course, there are many uses for flexures, and many flexure types. Flexure hinges, previously mentioned, are perhaps the most ancient. Straight-line motion mechanisms, arguably the most difficult bearing types to design, have reaped great benefit from flexure technology. The author spent 20 years designing and building bearings for scanning Michaelson interferometers, including diaphragm-flexure, "porch-swing" parallel-flexure, Bendix pivot porch-swing, and others. Because these straight-line motions are not generally considered pivots, they will not be discussed in detail here. The reader interested primarily in straight-line motions is recommended to References 4 and 5. Flexures of the torsional type are discussed in detail elsewhere in this book.

There is, however, a very interesting flexure pivot type whose design is due to the late Niels Young, and is illustrated in Figure 9.4. This pivot began life as a straight-line mechanism for a scanning Michaelson interferometer. The diaphragms, instead of being corrugated or plane, were pierced by a number of quasi-radial curved slots. These slots increased the axial compliance of the diaphragm considerably without materially decreasing the

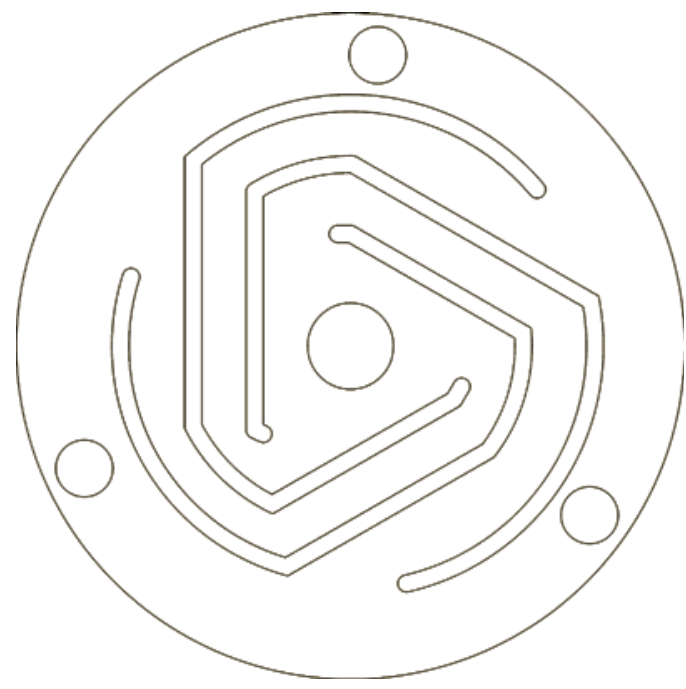

FIGURE 9.4

Support diaphragm for straight-line mechanism. 
radial stiffness, or introducing a departure from the straight-line motion. It was discovered, however, that if two of these diaphragms were mounted to their separating pillar with the slots aligned, a small rotation of the pillar accompanied translation. This was, of course, undesirable in the application, so the diaphragms were mounted reversed with respect to each other, and the rotation was restrained thereby.

Somewhat later the author having need of a very tiny precisely adjustable rotation in order to align an optical component, recalled the "defect" of the Young flexure, seized upon it as a virtue, and succeeded in putting it to good use.

Since the 1960s it has been generally recognized that the crossed-axis flexural pivot has the most widely applicable characteristics of any single-flexure pivot type. This wide adaptability was so compelling that the Bendix Corporation designed and manufactured a family of self-contained flexure pivots in various materials and sizes, and was so successful in its implementation that this type of pivot has come to be known worldwide as the Bendix pivot.

\subsubsection{The Bendix Pivot}

The Bendix "free-flex" pivot was introduced to the world in November 1962 in a seminal paper in Automatic Control magazine, entitled "Considerations in the Application of Flexural Pivots."'6

Available still from Lucas Aerospace, these high-quality, standardized, well-quantified pivots should be considered whenever there is space to include them, and the product envisioned will be produced in modest quantities. Much time and effort, particularly in qualification testing, can be saved by using these devices. Made in both single-ended (cantilever) and double-ended types, Figure 9.5 shows the general principle of construction. ${ }^{7}$

This general form of layout, the $90^{\circ}$ symmetrical cross, has become the standard of construction, and the departure point for many specialized designs. However, this layout has the defect that translation of the axis of rotation takes place during angular motion of the pivot. This motion is neither linear nor small, and while many optical applications

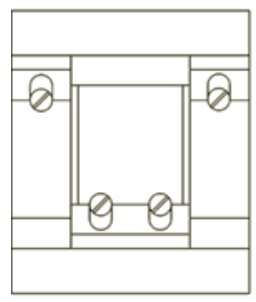

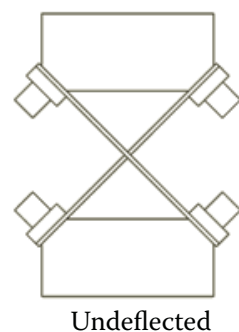

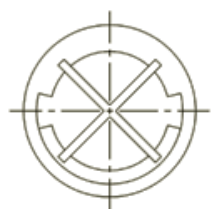

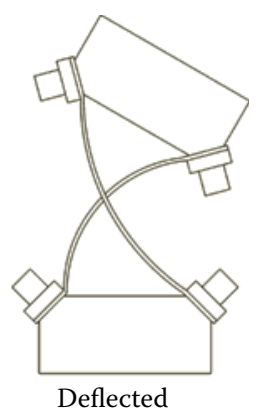

Deflected

FIGURE 9.5

The Bendix pivot. 
are quite tolerant of translation perpendicular to the plane of a mirror, the speed of the system may be limited by vibration produced by the translation of significant mass. As mentioned in the introduction to this chapter, it is possible to design a zero translation crossed-axis flexure pivot, and in principle possible to construct one, although the precision of assembly required is very high. The amount of translation, and the shape of the angle versus translation curve is dominated by the ratio of the leg lengths of the flexure. In the Bendix design, the leg lengths are equal, and the point of crossing, which lies on the axis of rotation, is initially central to the mounting tube. As the pivot is rotated, the axis of rotation departs from the center along a curve whose cusp is central and symmetric, but whose shape depends on the ratio of lengths of the flexure arms at intersection. Since the axis of rotation is usually a line fixed in space with respect to the rest of the mechanism, the axle is constrained by its load, and the flexures must cross on this axis, the only convenient way to change the ratio of the flexure arms is to extend the arm from the axis toward the stator (leaving the arm length between the axis and the rotor attachment point fixed) until the desired arm ratio is obtained. This inevitably has the effect of increasing the diameter of the stator flexure attachment point with respect to the axis of rotation. Since the ratio of arm lengths required for theoretical zero translation is $12.5 \%$ to $87.5 \%$, this can become a big deal, so most designs find a compromise that is workable.

\subsubsection{Cambridge Technology Crossed-Flexure Design Example}

Figures 9.6 through 9.9 show the successive stages of assembly of a flexure-pivot optical scanner mechanism, which represents the state of the art at Cambridge Technology.

This scanner, designed in 1995 and still in production, is used in a high-quality largeformat printing engine to produce multicolor magazine illustrations. It produces $30^{\circ}$ optical scans of an elliptical 30-mm aperture mirror at $160 \mathrm{~Hz}$, with line straightness of a few

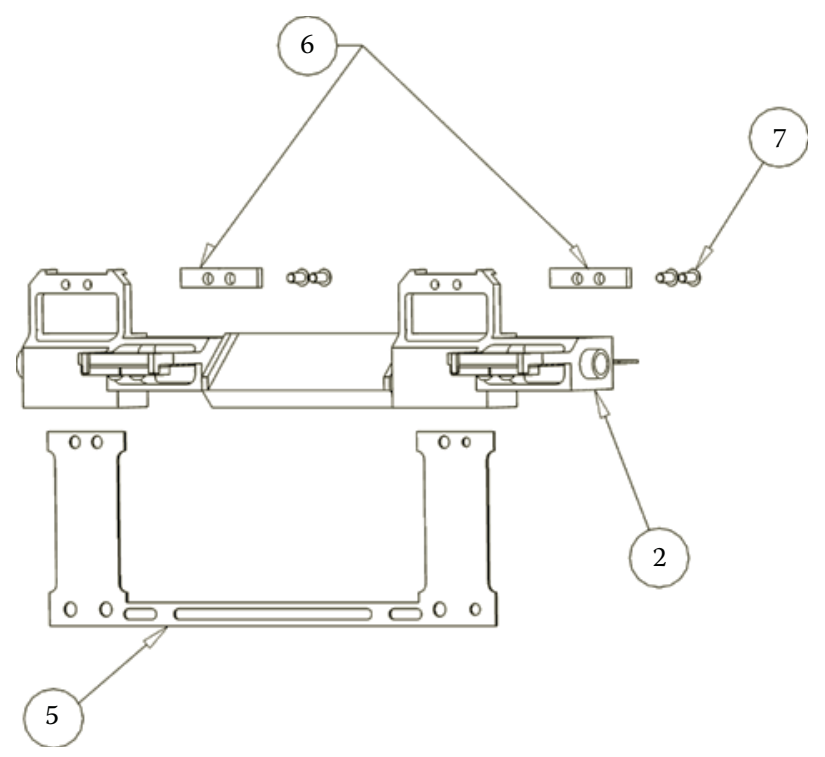

FIGURE 9.6

Rotor-flexure assembly. 


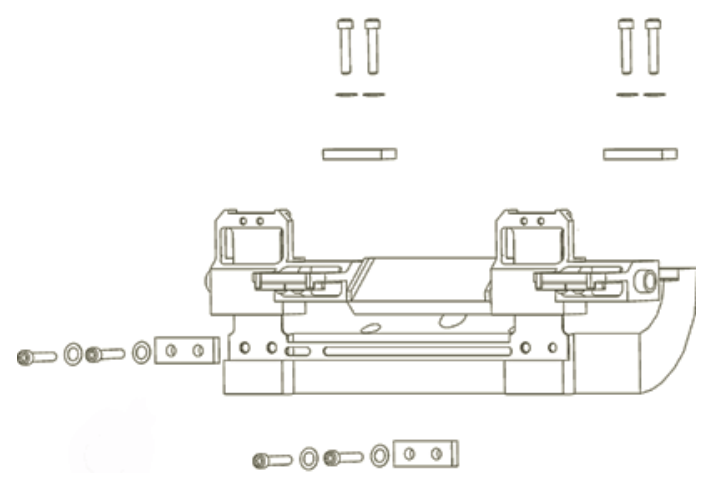

FIGURE 9.7

Rotor-stator assembly.

microradians. Every feature of design and construction highlighted in this text as preferred is illustrated in the design. Of the several thousand of these scanners delivered, of which many have more than $20,000 \mathrm{~h}$ of operation at $160 \mathrm{~Hz}\left(4 \times 10^{9}\right.$ cycles $)$, none has ever been returned for any reason.

Figure 9.6 shows the assembly of the flexures to the rotor. Note the radii in the mounting transition region of the flexures, the tie bar 5, and the lapped loose clamps 6 under the screw heads. These flexures are photo-etched, tumbled, 100\% edge inspected under 20x magnification and tin plated. Figure 9.7 shows the assembly of the rotor and its flexures into the stator. There are registration notches on the mounting pads on both the stator and the rotor, which position the ends of the flexures and their clamps, assuring equal effective length of the flexures and parallelism between the axis of the rotor and the axis of the stator. Notice that the flexure mounting pads are islands, which can be machined, lapped, and inspected easily. Once assembled to the stator sector, the entire pivot mechanism is complete, and can be inspected and tested easily without unnecessary obstruction. Once qualified, it is mounted into the final housing, such as the one shown in Figures 9.8 and 9.9.

An interesting variation of this design is illustrated in Reference 8. This scanner was required to operate in a mechanism that was quite sensitive to microscopic levels of vibration and other mechanical noise. Since crossed-axis pivots operated at high speeds produce a periodic translation of the axle, they have the potential to transmit vibration to the rest of the mechanism through their mounting means. In this case, the stator-rotor assembly, instead of being bolted directly to its housing, is supported on a set of flexures that permit an additional small rotation between the stator and the mounting. These flexures allow the pivot assembly to oscillate torsionally and transversely with respect to the housing. The second flexures therefore isolate the pivot assembly from the housing, and allow the housing to be bolted to the optical system without imparting an undesirable level of vibration in it. The second set of flexures allow the stator to rotate in response to the reaction torque from the rotor. The rotor and the stator counterrotate, and the relative amplitude of the angular oscillations of the rotor and the stator is approximately in inverse proportion to their respective inertias. A typical ratio of inertias for the rotor and the stator is 1:150, so that the angular deflection of the second set of flexures is approximately $1 / 150$ as great as that of the rotor flexures. The second set of flexures also allows the stator to translate in response to the oscillatory translations of the rotor's center of 


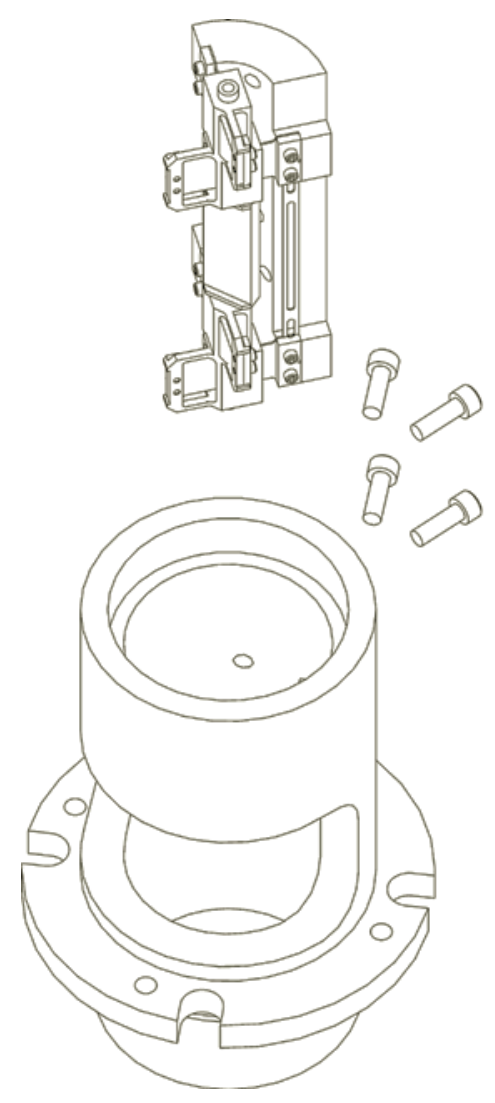

\section{FIGURE 9.8}

Stator-housing assembly, exploded view.

gravity, which result from the translation of the rotor as it rotates. The forces required to dynamically accelerate the rotor and the stator largely balance each other. The amplitude of the residual translation force, compared with the translation force of the same pivot assembly bolted directly to the housing, is given approximately by the ratio of the pivot assembly mass to the housing mass, typically 1:15 or greater. One could, of course, apply ever more stages of isolation by this means of flexure pivots within flexure pivots if one had the need to do so.

\subsection{LOW-COST CANTILEVER SCANNER}

The discussion of the previous section was devoted to the details of construction of a typical very high performance scanner where the unique attributes of the flexure pivot are devoted to solving issues unmanageable by any other known bearing type. On the opposite end of the spectrum of suitable applications, flexure pivots lend themselves to the construction of very low-cost scanners which yet are rugged, long-lived, and precise. 

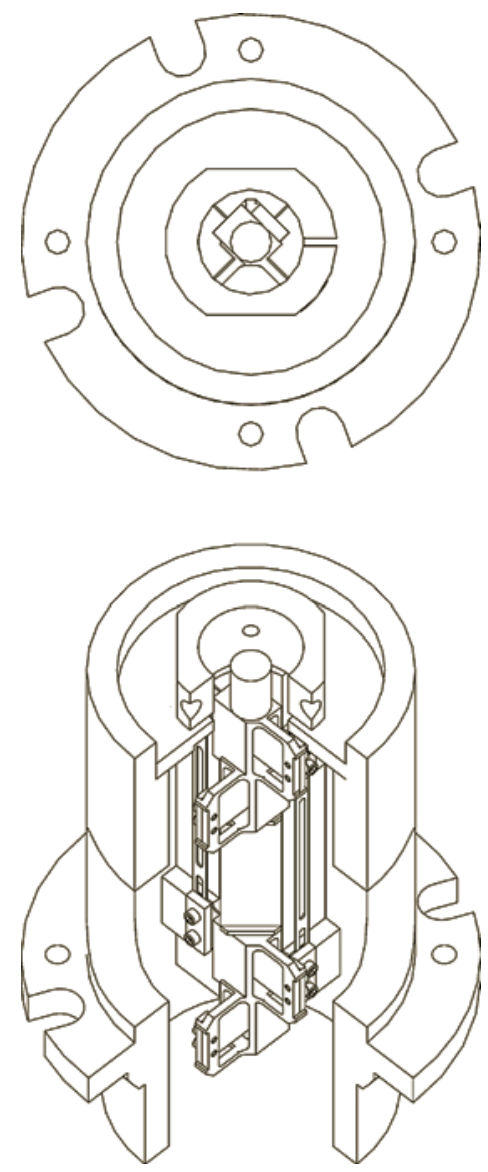

FIGURE 9.9

Final scanner assembly.

\subsubsection{General Features}

Figure 9.10 shows a cantilever flexure scanner designed for a digital radiography application. It operates at $50 \mathrm{~Hz}+/-1 \mathrm{~Hz}$ over a peak-to-peak optical scan angle of $40^{\circ}$. The mirror aperture is designed to accommodate a 12-mm diameter beam at a nominal angle of incidence of $45^{\circ}$. Figure 9.10 shows a plan and an elevation view of the scanner. Figure 9.11 shows a picture of the flexure itself. Figure 9.12 shows an exploded view, where 1 is the flexure, 2 is the mirror, 3 is the flexure clamp, 4 is the flexure support, 5 is a counter mass, and 6 is the drive magnet. Referring now to Figure 9.10, the elevation view has an arrow which indicates the motion of the mirror. Cantilever scanners pivot the mirror a long way from the center, and so the mirror "walks" on the beam during the scan; the cross-axis dimension of the mirror is long. The elastic curve of the flexure depends on the way the drive is applied. In this particular design, the drive is by means of a small magnet attached under the center of the mirror at the tip of the effective length of the flexure. The magnet dips into a pair of coils in the stator block, wound at $90^{\circ}$ to each other and mutually at $45^{\circ}$ to the axis of the magnet. One of the coils provides the drive flux, and the other serves as a velocity coil for feed back. (This coil construction is intended to minimize the transformer coupling between the drive and feed back coils.) This kind of drive coupling 


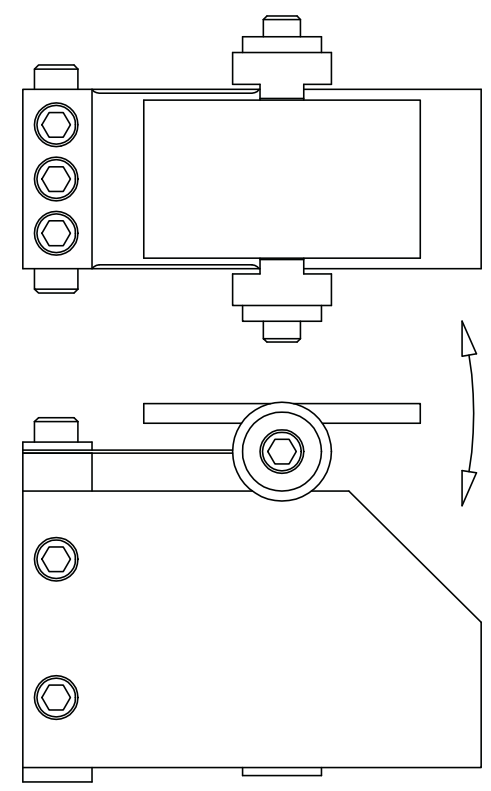

\section{FIGURE 9.10}

Low-cost cantilever scanner.

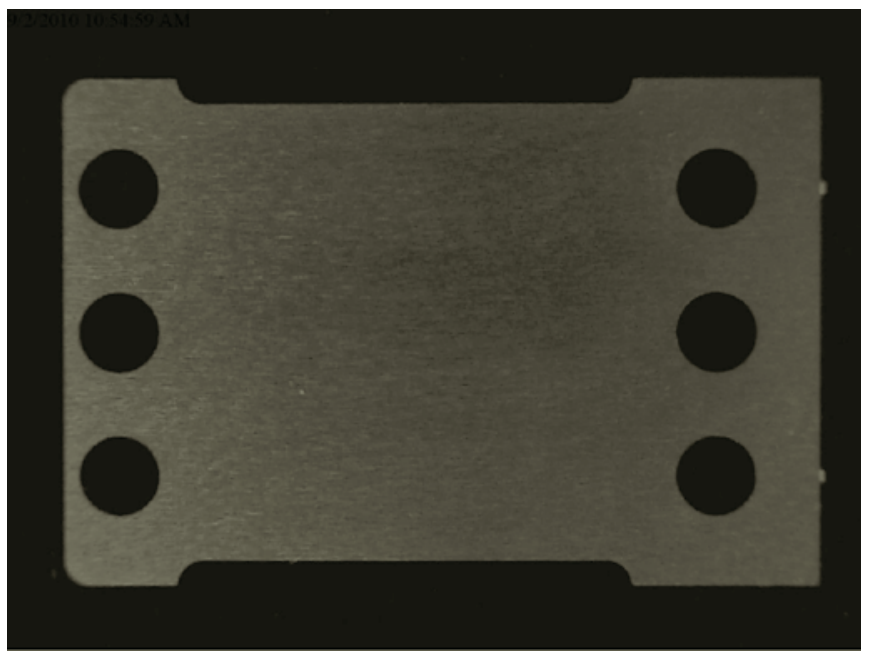

\section{FIGURE 9.11}

Flexure.

is a combination of point loading at the tip and loading as a moment around the tip. As a result, the slope of the tip of the flexure, and therefore the slope of the mirror surface does not obey either the $-P L^{*} 2 / 2 E I$ or the $-M L / E I$ textbook expressions, but instead a combination, which is sensitive to the exact relationship between the magnet and the back iron which makes prediction of the slope uncertain. Of course, other more predictable drive 


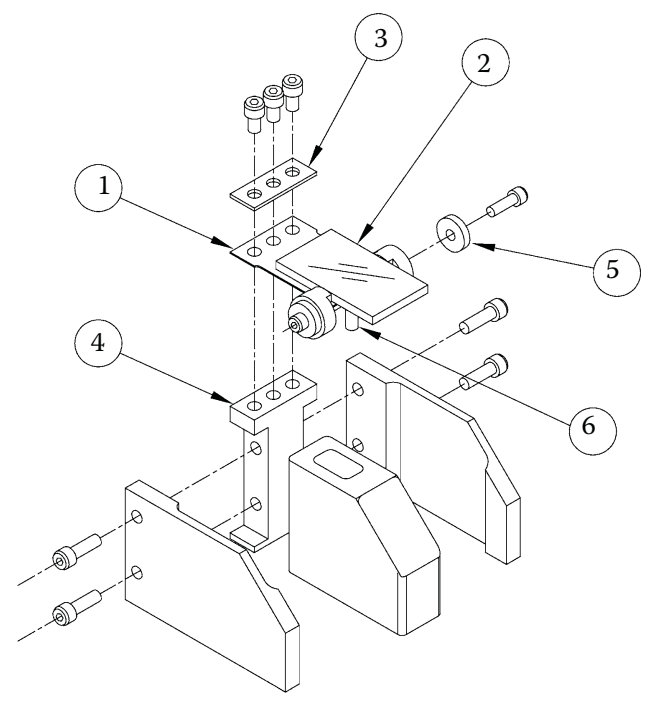

FIGURE 9.12

Cantilever scanner, exploded view. 1. Flexure, 2. Mirror, 3. Clamp, 4. Mounting post, 5. Tuning weight, 6. Drive magnet.

coupling methods are possible if prediction of the slope is important to the design. In any case, the fact that the flexure assumes a curve has the effect of reducing the magnet deflection required for a particular scan angle compared to that of a simple hinge, and this means that the peak stress, which occurs at the clamped end of the flexure, is reduced. It also means that, while the motion of the mass-spring is sinusoidal, the motion of the deflected beam is partially linearized. In fact one could depart from the simple constantsection rectangular flexure in favor of a triangular flexure, or a rectangular flexure tapered in thickness in the form of a cubical parabola, both of which have as their elastic curve a circular arc, and more completely linearize the velocity of the reflected beam. Both of these variations have complexities which increase the cost, and so are inappropriate for a design which is intended, as this one is, to be low cost.

\subsubsection{Design example}

It is useful to begin with the inertia of the moving parts, since in general the size of the mirror and the scan angle are defined a priori. The essential moving parts are the mirror and the drive coupling, in this case a magnet. The inertia of the flexure may usually be ignored; however, including one-third of the flexure inertia produces a rigorous result. There are usually ancillaries to the required rigid connection between the flexure, the mirror, and the drive which add inertia, in this case an aluminum spool, a flexure clamp (not shown, but essentially identical to part 3), and three small screws. In addition, this design includes provision for tuning weights. This is not essential, because conventional dimensional control of the flexure and moving parts, once the desired frequency is established empirically, is adequate to assure high yield in production even with fairly tight frequency specification $(+/-2 \%$ in this case). However, the provision of tuning weights means that the empirical frequency "tweak" is straightforward, and furthermore, the weights permit the 
basic scanner to be configured for a variety of other mirror sizes and resonant frequencies simply by changing the weights at final assembly.

\subsubsection{Motor Size Required}

Assuming a conservative mechanical " $Q$ " of 1000, the motor will need to deliver about $1 / 1000$ of the stored energy per cycle. The stored energy is given by $U=f d / 2$. As pointed out above, we don't know exactly what the value of the displacement will be, but a conservative estimate is given by ignoring the fact that the flexure will bend in an arc, and assume a deflection given by the multiplying the length of the active part of the flexure times the peak scan angle in radians. Let us assume a flexure length of $0.5 \mathrm{in}$, a spring rate of $6 \mathrm{lb} / \mathrm{in}$, and a peak mechanical angle of $10^{\circ}$ or 10/57.3 rad. The displacement is then 0.5 $\times 10 / 57.3=0.087 \mathrm{in}$, the force is $0.522 \mathrm{lb}$, and $\mathrm{U}=8.3 \times 10^{-2} \mathrm{in} \mathrm{lb}$. The minimum motor size required then will be such as to supply $8.3 \times 10^{-2} / 1000=8.33 \times 10^{-5} \mathrm{in} \mathrm{lb}$ to the drive. To put this all in perspective, the power of the minimum-size motor in this case is $8.33 \times 10^{-5} \times 50$ $=4.2 \times 10^{-3}$ in lb/s or $1.3 \times 10^{-7}$ Watts. Inevitably there are $I^{*} 2 R$ losses and windage losses, and the scan angle will take some time to build up with a minimum-size motor, so it is usual to make the motor somewhat oversize. That said, it is evident that such low-power scanners make possible long-life battery powered equipment.

\subsection{VIBRATING-WIRE SCANNER}

Figure 9.19 shows the general lay out of a vibrating wire scanner. Using the expression $F=\sqrt{ } T / L W$, well known to string musicians, and dating from around the year 1300 we can design a scanner to use two strings vibrating $180^{\circ}$ out of phase to tip a mirror.

$$
\begin{aligned}
F & =\text { Desired frequency of vibration } \\
T & =\text { wire tension } \\
L & =\text { length of wires } \\
W & =\text { mass of moving parts }
\end{aligned}
$$

The spacing between the wires is arbitrary. The mirror is fastened to the midpoint of each wire as shown, and the drive is attached to the underside of the mirror between the wires. In general, the weight of the wires may be ignored, since the mirror and drive are the major source of mass, but again, adding one-third of the weight of the wires is rigorous. Both ends of the pair of wires are clamped, and some means of adjusting the tension of the wires must be provided. Rearranging the expression, $T=L F^{*} 2 W$ in appropriate units. The drive may be arranged as with the cantilever scanner, and all the " $Q$ " and power considerations described in that section apply. This scanner, with suspension support at both ends, is more resistant to vibration than the cantilever scanner, and is suitable for a wide range of operating frequencies, just like the Piano.

\subsection{MICROELECTROMECHANICAL FLEXURE SCANNERS}

Microelectromechanical scanners (MEMS) integrate flexures and electromechanical actuators to yield small scanners of high performance and low cost. These are interesting in a number of applications but none more than optical switching in telephony. 
The scanning mirror may be formed direct onto the MEMS carrier, or it may be a separate component bonded to the mechanical assembly. Its aperture is typically between $0.1 \times$ $0.1 \mathrm{~mm}$ and $3 \times 3 \mathrm{~mm}$. Currently, optical scan angles up to $20^{\circ}$ are practical. Resonant frequencies are typically in the range $10-40 \mathrm{kHz}$ and are dependent on system design, mirror size, and maximum scan angle.

There is a huge body of expertise in the manufacture of precise, small structures from silicon or on silicon substrates. It is fortuitous that silicon possesses attractive properties for small flexures, so we can exploit the existing semiconductor manufacturing infrastructure to make MEMS. This same manufacturing technology is well suited to the fabrication of the piezoelectric actuators.

\subsubsection{MEMS Design}

Piezoelectric bimorphs consist of two strips of piezoelectric elements joined over their long surfaces, provided with electrodes in such a manner that when an electric field is applied, one strip elongates and the other contracts. This results in a bending motion of both strips. The motion of the tip can be considerable, which is what makes these devices such useful tools as converters of electrical energy to mechanical energy and vice versa. They are used in different applications such as ultrasonic motors, ${ }^{9}$ laser beam detectors, ${ }^{10,11}$ fans for cooling electronics, ${ }^{11}$ numeric displays, ${ }^{12}$ filters, ${ }^{13}$ accelerometers, ${ }^{14-16}$ optical choppers, ${ }^{17}$ and more recently as the legs of microrobots. ${ }^{18}$ They are suitable as converters of electrical signals into sound (speakers) and similarly as pickup elements for the detection of sound. ${ }^{19}$ They are also used as the control element to reduce vibration in space-borne structures such as solar panels and in the walls of offices for the reduction of sound transmission. ${ }^{20}$ While there is a huge variety of concepts that could be realized, we confine this discussion to a relatively simple layout as represented in Figure 9.13.

The two piezoelectric actuators are of a form that has been used for the last 70 years or more. ${ }^{9}$ This "bender," as we shall refer to it, has various names in the literature, including "piezoelectric bimorph," a term not strictly accurate in this case. It comprises two bars of

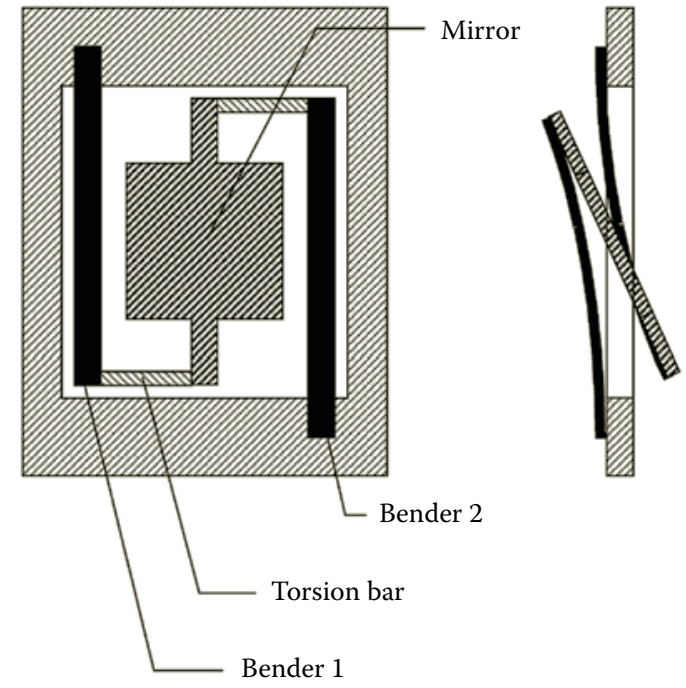

FIGURE 9.13

Simple piezoelectric "bender" scanner. 
piezoelectric material, typically PZT, having opposite polarities and sandwiched between two electrodes as indicated in Figure 9.14. A voltage applied to the electrodes results in a field that causes the length of one bar to increase and that of the other to reduce. This produces curvature of the structure similar to the bending of a bimetal structure as a result of a temperature change. In our case, the benders are, strictly speaking, monomorphs; that is, there is only one active layer of piezoelectric material.

The free ends of the two benders are connected by torsion bars to the mirror substrate. The benders are driven by equal and opposite polarity so their motion is equal and opposite. The mirror substrate rotates about a central axis. This geometry has been studied in depth by Smits.

When using PZT material, an electric field is applied across two plates separated by poled ferroelectric material. PZT ceramics are isotropic and not piezoelectric prior to poling. Upon poling, they become anisotropic and display directionally dependent piezoelectric and mechanical properties. Poling is accomplished by heating the material to its Curie temperature and applying a DC field to the crystal, which then aligns its previously randomly oriented dipoles parallel to the field. Upon cooling, the dipoles maintain this preferred arrangement. As a result, there is a crystal distortion that causes growth in the dimension parallel with the field and also in the dimension perpendicular to the field. The axial strain resulting is typically small $(0.2 \%)$ and is accompanied by hysteresis.

The displacement of the PZT material in the actuators when an electric field is supplied is the source of the bending moment of the " $\mathrm{J}$ " arms connected to the mirror platforms. When the electric field is applied, the material has displacement in two directions: parallel to the field and at a right angle to the field. The parallel and perpendicular displacements are opposite in sign so that when the film expands in parallel with the field it contracts its dimension perpendicular to the field and vice versa. The polarity of the parallel displacement is determined by the direction of the electric field with respect to the domains of the material. (The directions of the domains were established during the polarization process.) If the polarization and electric field are opposed, that is, the domains are in the opposite direction to the applied electric field, the material will expand parallel to the field and shrink perpendicular to the field. Opposed domains are stable in the sense that they are already arranged to cancel the applied electric field. If the domains and electric field are parallel, then the material will shrink as the electric field gets stronger until $50 \%$ of the domains have switched their direction to be opposed. This is effectively repolarizing the

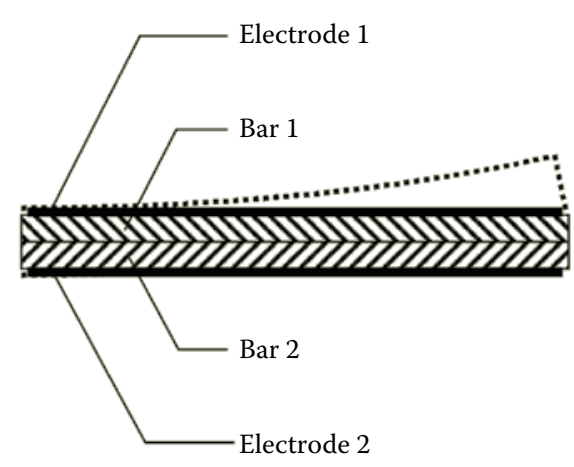

FIGURE 9.14

Piezoelectric "bender" structure. 
material. When more than $50 \%$ of the domains have switched, the material will begin to expand again.

The amount of displacement caused in the case of the opposed situation can be estimated by the following formula:

$$
D_{3}=+d_{33} \times\left(V_{3}\right)
$$

where $D_{3}$ is the displacement in the " 3 " direction, (the " 3 " direction is perpendicular to the capacitor plates), $V_{3}$ is the applied voltage between the plates of the capacitor, and $d_{33}$ is the coefficient for displacement parallel to the field, typically $7 \times 10^{-12} \mathrm{~m} / \mathrm{V}^{2}$.

\subsubsection{MEMS Manufacture}

Exploitation of the existing silicon fabrication technology leads naturally to the manufacturing process represented by Figure 9.15 . Each MEMS module is typically $4 \times 4 \mathrm{~mm}$. The modules are produced in arrays on a silicon wafer to yield 50 to several hundred modules per wafer.

The correct operation of the MEMS depends on symmetry of the performance of the two benders in the assembly. This symmetry and repeatability of performance from module to module are achieved only through precise dimensional, process, and material control.

The fabrication process is as follows:

1. A silicon wafer with a buried oxide layer is oxidized (Figure 9.15a).

2. Front- and backside alignments are applied in photoresist (not shown).

3. The wafer is etched in BOE (buffered oxide etch), removing the oxide layer, leaving identical marks on front- and backside (Figure 9.15c).

4. The wafer is etched in $\mathrm{NaOH}$ leaving a protruding pyramid at the backside and an inverted pyramid on the front side. (Figure 9.15c).

5. The wafer is reoxidized (Figure 9.15d).

6. The backside is now etched to depth of 350 microns, which leaves a varying thickness of the membrane on the front side, according to the location of the point where the measurement is made. A Pt under electrode is deposited and PZT is applied as a sol-gel. A Pt top electrode is deposited (Figure 9.15f).

7. On the front side the outline of the double monomorph optical scanner structure is etched out to the depth of the insulating buried oxide layer, while the backside is protected (Figure 9.15g).

8. The wafer is reoxidized (not shown).

9. 25 microns is removed from the backside, to etch free the double monomorph optical scanner structure. The etching stops at the buried oxide layer (Figure 9.15h).

Figure 9.16 shows a schematic of a double monomorph optical scanner. Figure 9.17 shows an SEM photograph of a scanner at 50× magnification.

\subsubsection{Operation of the Scanner}

In Figure 9.18 a cross section is given, in which the eye is located in the plane of the wafer. The angle $\beta$ is the angle the mirror makes with the plane of the wafer. 
(a)

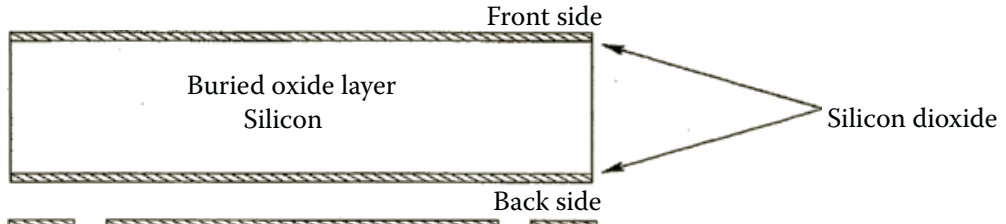

(b)

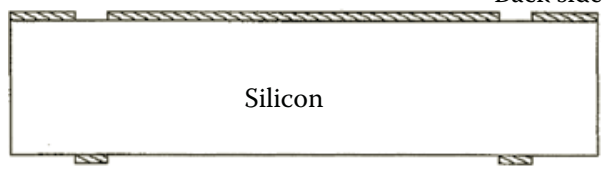

(c)

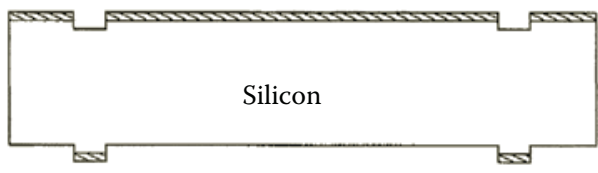

(d)

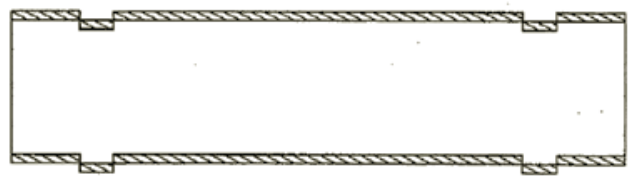

(e)

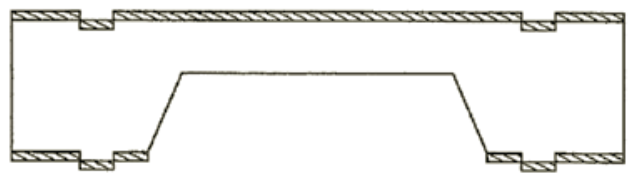

(f)

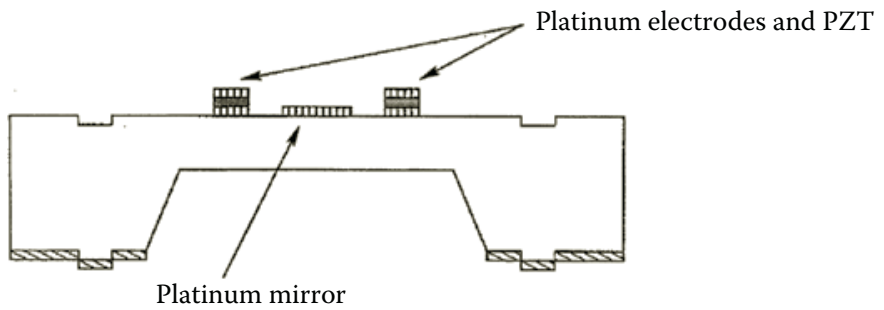

(g)

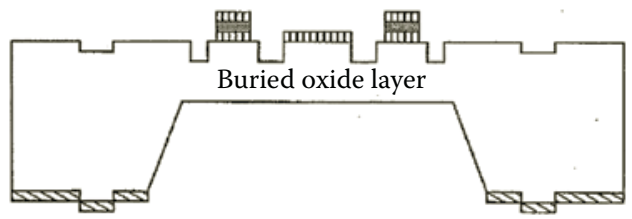

(h)

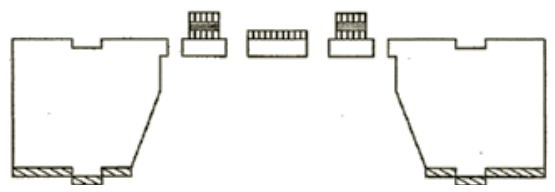

FIGURE 9.15

An illustration of the fabrication process. (a) Oxidized silicon blank, (b) First etch, (c) Second etch, (d) Second oxidation, (e) Third etch, (f) Deposition step, (g) Fourth etch, (h) Final etch. 


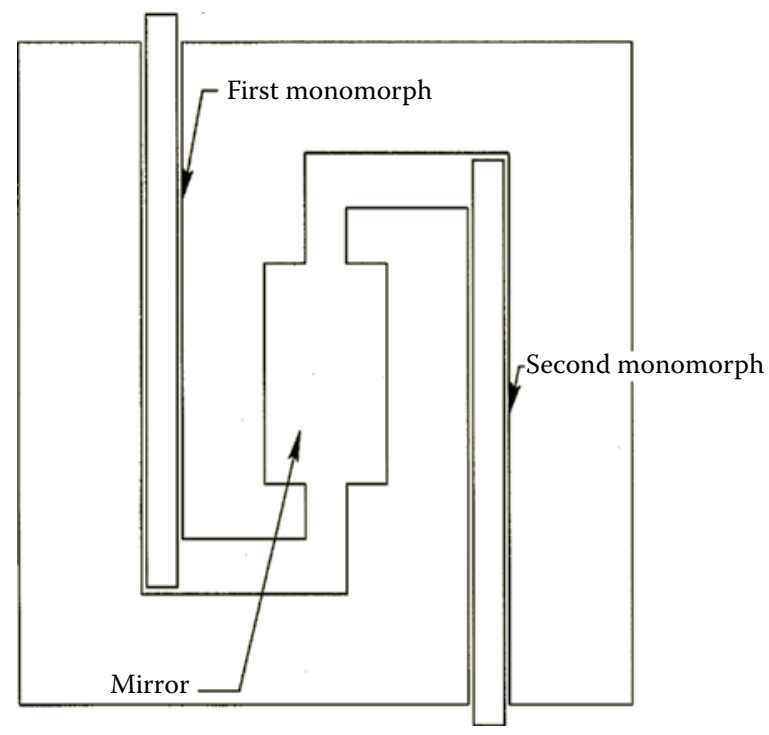

FIGURE 9.16

Schematic of double monomorph optical scanner.

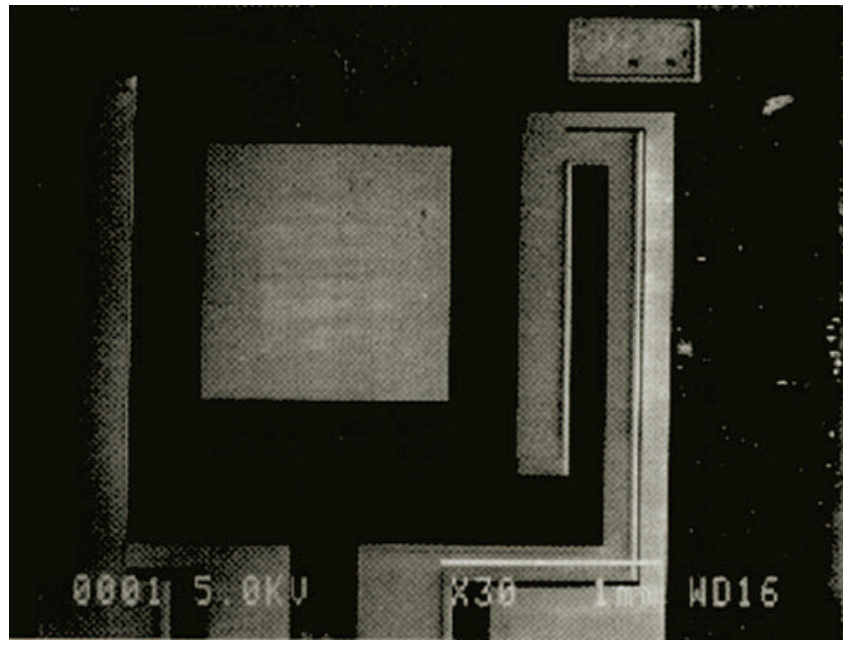

FIGURE 9.17

$5 \times$ SEM photograph of MEMS scanner.

\subsubsection{Material Properties}

The properties required of the driving materials are a reasonably high coefficient of strain versus applied field, low or predictable hysteresis, and high repeatability. The properties required of the support materials are ease of fabrication, very high stability, and a high fatigue limit. These are met satisfactorily in the silicon mechanical material chosen: it was in fact, these demonstrated characteristics of silicon that led to its choice as a basis material. On the other hand, while the PZT material has a satisfactory coefficient of strain, these constraints remain. Although these materials are excellent for submicron positioning, they 


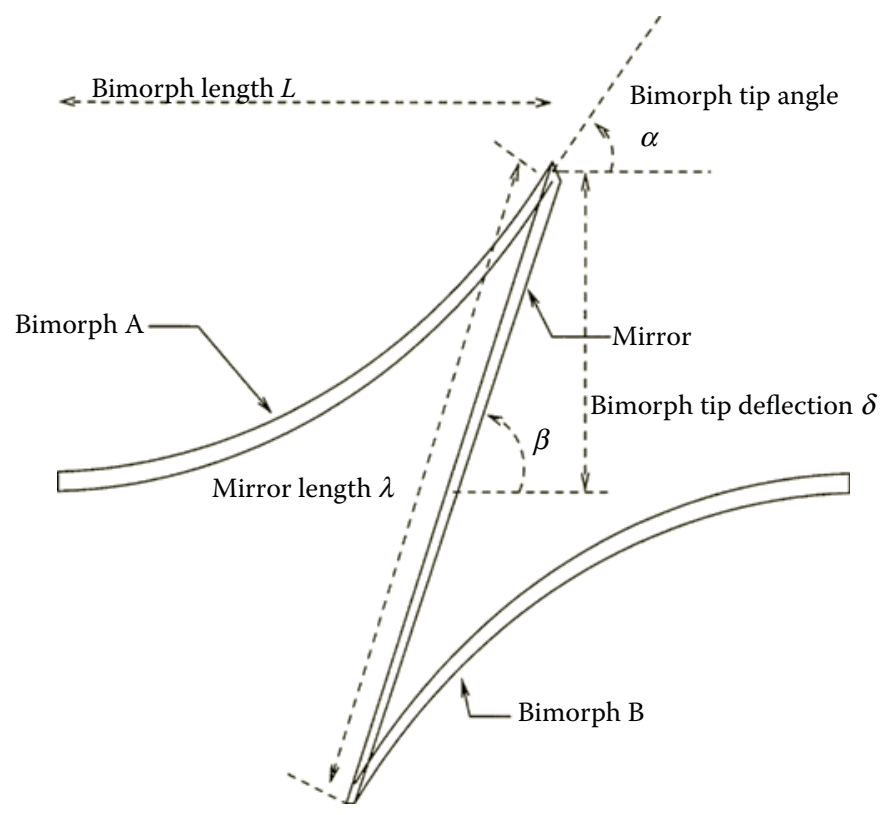

FIGURE 9.18

Cross-sectional schematic of double monomorph optical scanner.

have inherent hysteresis and creep, and so they lack the level of repeatability required in a practical open-loop positioning device. Long-term creep can approach 15\%, and hysteresis can approach $12 \%$.

\subsubsection{Static Performance}

\subsubsection{Hysteresis}

Hysteresis appears as apparent "backlash" upon reversal of the direction of motion, but unlike the backlash of conventional mechanical systems and stiction, which can largely be predicted and compensated, hysteresis depends on the recent history of motions and is difficult to model, predict, and compensate. The creep is, at this time, completely unpredictable. As a result, open-loop piezoelectric devices are limited to applications in which repeatability of $10 \%$ is satisfactory. Of course, when combined with a position feedback system, this defect can largely be overcome, but the cost of this level of control is disproportionate to the economics of the production of MEMS.

\subsubsection{Linearity}

Linearity of motion over mirror angles of $10^{\circ}$ mechanical is in the range of $2 \%$, smoothly changing, monotonic, and predictable. This characteristic is considered satisfactory for most laboratory uses. However, PZT has a rather large strain sensitivity to temperature, so that linearity of motion can be compromised by changes in temperature.

\subsubsection{Uniformity}

Uniformity of performance scanner to scanner within a wafer is entirely a question of process control, and is not expected to pose long-term problems. Standard levels of production 
process control are also expected to address wafer-to-wafer performance uniformity issues satisfactorily. At the moment, we are achieving uniformity of characteristics of about $10 \%$ across the wafer and wafer to wafer in a pilot production phase.

\subsubsection{Yield}

Yield is an issue that falls into the process control purview as well. At the moment, we are achieving yields above $80 \%$, with most of the dropouts the result of mirror defects.

\subsubsection{Dynamic Performance}

\subsubsection{Dynamics}

These scanners have a first torsional resonance typically well above $20 \mathrm{kHz}$ with integral mirrors. As a result, the dynamics permit full-scale $\left(10^{\circ}\right)$ steps in well under $100 \mu \mathrm{s}$. With high-quality mirror flakes 150 microns thick cemented over the integral mirror, the resonant frequency falls to about $8 \mathrm{kHz}$, which is satisfactory for most microscanning purposes. The flatness of the present examples of integral mirrors is poor, as is the surface finish, but attached flakes achieve any required surface quality. Testing has verified that 150 micron thickness is adequate to preserve quarter wave or better flatness over the $1.8 \times$ 2.6 aperture of the standard scanner because of the underlying silicon support ring.

\subsubsection{Life}

Life testing has revealed no observable damage after $10^{10} 60^{\circ}$ optical scans when driven by an $8-\mathrm{kHz}$ oscillator. Flexure stress is expected to be below the endurance limit for silicon at all angles of operation up to $30^{\circ}$ mechanical.

\subsubsection{Degradation Processes}

No degradation process is known, other than excessive voltage breakdown inside the PZT crystal, and heating above the Curie point. High-speed operation (above $20 \mathrm{kHz}$ ) has the potential to encounter impact degradation of the tips of the bender arms and the edges of the mirror if operated in open air. The standard packaging presently envisioned for individual scanners is a hermetically sealed TO5 package.

\subsubsection{Application Rules}

\subsubsection{When and When Not to Use MEMS}

The current state of the art in PZT open-loop scanners has limited repeatability and "staring" positional stability because of creep and hysteresis. As a result, MEMS scanners should not be specified for applications in which positional precision better than $10 \%$ of full scale is required unless some form of active position feedback is anticipated. On the other hand, the device behaves as though it were a pair of capacitors of a few nanofarads capacity. Therefore, self-heating even during aggressive scan profiles is not an issue, and cooling is not required except in environments above $100{ }^{\circ} \mathrm{C}$. Because the thermal transport path from the mirror through the structure is long, the effect of power coupled from the scanned beam may be significant. In this case, it is desirable to provide a direct mirror cooling path through a suitable gas, such as helium, directly to the scanner case. 
Vibration and inertial forces applied to the scanner are likely to cause deformation of the flexure structure, and, since the system is very poorly damped, are likely to produce long-lasting settling periods (Figure 19.9). Otherwise, these scanners have no particular susceptibility to environmental stimuli such as temperature, barometric pressure, humidity, magnetic and electric fields, and so forth.

\subsubsection{Anticipated Developments}

It is anticipated that electrostrictive actuators may replace piezoelectric actuators in MEMS in the near future. A typical electrostrictive material, such as lead-magnesium-niobate (PMN), should provide an order of magnitude improvement over PZT in positional stability.

For PMN materials, the change in length is proportional to the field voltage squared, so the coefficient is a factor of two higher than PZT. Unlike piezoelectric materials, PMN crystals are not poled. Positive or negative voltage changes result in an elongation in the direction of the applied field, regardless of its polarity. Because PMN is not poled, it is inherently more stable than PZT, resulting in a reduction of long-term creep from $15 \%$ to $3 \%$. Also, PMN materials have better hysteresis than does PZT. While PZT exhibits 12\% hysteresis, PMN displays only $2 \%$.

Two properties of PMN contribute to its thermal stability. First is the strain sensitivity to temperature. The PMN is much more robust than PZT in this regard, especially over large temperature ranges. Secondly, the coefficient of expansion of PMN is twice that of PZT.

\subsubsection{Conclusions}

It is clear that MEMS have not yet grown from infancy. Unlike their mature brethren, the macroscale flexure scanners, the only conclusion one could legitimately draw at this time is that the future is bright for small scanning and pointing devices that draw little power, pack closely together, demonstrate extreme reliability, and offer a price-performance index beyond the capability of any macroscale device. It seems certain that these attributes of MEMS will be exploited. Just how MEMS will look in their maturity years hence is anyone's guess.

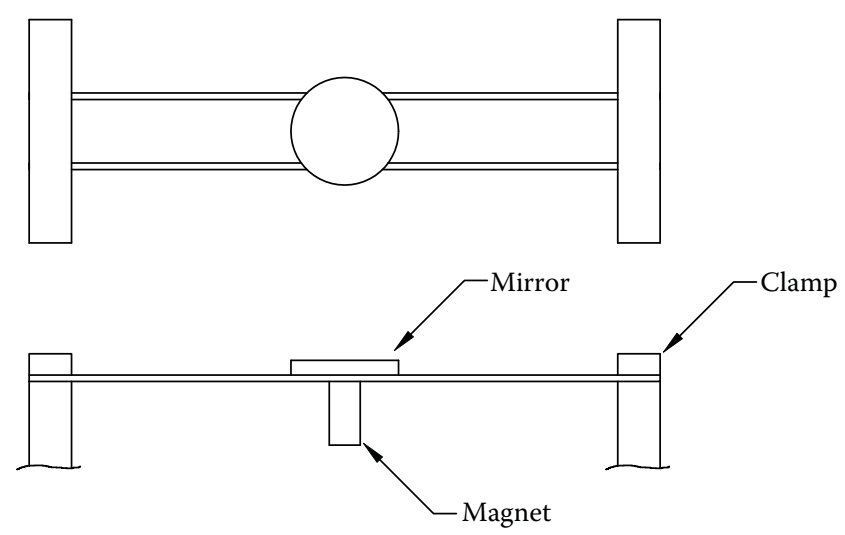

FIGURE 9.19

Vibrating wire scanner. 


\subsection{CONCLUSION}

It seems that flexure pivots have come of age. They have been used in a great diversity of products where their attributes of sensitivity, accuracy, and repeatability are enabling. They are also low cost, lubricant-free, low mass, high " $Q$," and capable of storing considerable energy. They can be cascaded to provide vibration isolation. There are standalone commercial versions to suit many purposes. The published data on detail design rules are considerable, and no competent engineer should have unusual difficulty in producing a workable pivot on the first try. It is the hope of the authors that the information in this chapter will help neophytes to avoid most of the impediments to success that lurk in the mysteries of material selection, processing, and the mounting of flexures and that many more useful applications of flexure pivots will be found and pursued with success.

\section{ACKNOWLEDGMENTS}

First of all, may I thank my friend and colleague, Felix Stukalin, without whose encouragement and forbearance this chapter would not have been written at all. Brian Stone performed the labors of Hercules in turning my sketches and doodles into the illustrations. Michael Nussbaum read the manuscript, and made many helpful suggestions. Dr. Tim Weedon and Reggie Tobias were both very perceptive as well as diplomatic in their criticisms. Last but not least, Professor Jan Smits did all the heavy lifting in the second part of the chapter. Assisting him in his laboratory at Boston University, Koji Fujimoto and Vladimir Kleptsyn did much of the MEMS construction and testing, and Steven Vargo and Dean Wibig of JPL, and Joe Evens and Gerry Velasquez of Radiant Technologies Inc. provided enabling processing services.

\section{REFERENCES}

1. Weinstein, W.D. Flexure pivot bearings. Machine Design 1965, 37, 136-145.

2. Bendix flexural pivot. Bendix Electric and Fluid Power Division, Application Notes, Catalog, Bendix Corp., Utica, NY.

3. Sines and Waisman, Eds. Metal Fatigue; McGraw Hill 1959, 89-111.

4. Boyer, H.E., Ed. Failure analysis and prevention. In Metals Handbook, 8th Ed., Vol. 10; American Society for Metals: Metals Park, OH; 208-249.

5. Paros, J.M.; Weisbord, L. How to design flexure hinges. Machine Design 1965, 37, 151-156.

6. Neugebauer, G.H. Designing springs for parallel motion. Machine Design 1980, 52, 119-120.

7. Troeger, H. Considerations in the application of flexural pivots. Automatic Control 1962, 17(4), 41-46.

8. Paulsen, D.R. Flexural Pivot. U.S. Patent 4,802,720, February 7, 1989.

9. Brosens, P.J. Resonant Optical Scanner. U.S. Patent 5,521,740, May 28, 1996.

10. Sawyer, C.B. The use of Rochelle salt crystals for electrical reproducers and microphones. Proc. Inst. Radio Eng. 1931, 19(11), 2020-2029.

11. Smits, J.G.; Dalke, S.I.; Cooney, T.K. The constituent equatons for piezoelectric bimorphs. Sensors and Actuators 1991, 28, 41-61. 
12. Kugel, V.D.; Xu, B.; Zhang, Q.M.; Cross, L.E. Bimorph based piezoelectric air acoustic transducer: A model. Sensors and Actuators.

13. Caliano, G.; Lamberti, N.; Iula, A.; Pappalardo, M. A piezoelectric bimorphstatic pressure sensor. Sensors and Actuators A 1995, 46(1-3), 176-178.

14. Coughlin, M.F.; Stamenokic, D.; Smits, G. Determining spring stiffness by the resonance frequency of cantilevered piezoelectric bimorphs. IEEE Trans. Ultrasonics, Ferroelectrics and Frequency Control 1977, 44, 730-733.

15. Kielczynski, P.; Pajenski, W.; Salewski, M. Piezoelectric sensors for the investigation of microstructures. Sensors and Actuators A 1998, 65(1), 13-18.

16. Juan, I.; Roh, Y. Design and fabrication of piezoceramic bimorphvibration sensors. Sensors and Actuators A 1998, 69(3), 259-266.

17. Van Mullem, C.J.; Blom, F.R.; Fluitman, J.H.J.; Elwenspock, M. Piezoelectrically driven silicon beam force sensor. Sensors and Actuators A 1991, 26(1-3), 379-383.

18. Naber, A. The tuning fork as a sensor for dynamic force control in scanning near-field optical microscopy. J. Microscopy-Oxford 1999, 194(2-3), 307-331.

19. Yamada, H.; Itoh, H.; Watanabe, S.; Kobayashi, K.; Matsushige, K. Scanning near-field optical microscopy using piezoelectric cantilevers. Surface and Interface Analysis 1999, 27(5-6), 503-506.

20. Kielczynski, P.; Pajewsli, W.; Sealcwski, M. Piezoelectric sensor applied in ultrasonic contact microscopy for the investigation of material surfaces. IEEE Trans Ultrasonics, Ferroelectrics and Frequency Control 1999, 46(1), 233-238.

21. Edwards, H.; Taylor, L.; Duncan, W.; Melemed, A.J. Fast, high-resolution atomic force microscopy using a quartz tuning fork as actuator and sensor. J. Appl. Phys. 1997, 82(3), 980-984. 